\title{
J-PLUS: A first glimpse at the spectrophotometry of asteroids The MOOJa catalog ${ }^{\star}$
}

\author{
David Morate ${ }^{1,5,6}$, Jorge Marcio Carvano ${ }^{1}$, Alvaro Alvarez-Candal ${ }^{2,3,1}$, Mário De Prá ${ }^{4}$, Javier Licandro ${ }^{5,6}$, \\ Andrés Galarza ${ }^{1}$, Max Mahlke ${ }^{7,8,9}$, Enrique Solano-Márquez ${ }^{8,9}$, Javier Cenarro ${ }^{10}$, David Cristóbal-Hornillos ${ }^{10}$, \\ Carlos Hernández-Monteagudo ${ }^{10}$, Carlos López-Sanjuan ${ }^{10}$, Antonio Marín-Franch ${ }^{10}$, Mariano Moles ${ }^{10}$, \\ Jesús Varela ${ }^{10}$, Héctor Vázquez Ramió ${ }^{10}$, Jailson Alcaniz ${ }^{1}$, Renato Dupke ${ }^{1}$, Alessandro Ederoclite ${ }^{11}$, \\ Laerte Sodré Jr. ${ }^{11}$, Raul E. Angulo ${ }^{12,13}$, Francisco M. Jiménez-Esteban ${ }^{8,9}$, \\ Beatriz B. Siffert ${ }^{14}$, and J-PLUS Collaboration \\ 1 Observatório Nacional, Coordenação de Astronomia e Astrofísica, 20921-400 Rio de Janeiro, Brazil \\ e-mail: davidmorate@on.br \\ 2 Instituto Universitario de Física Aplicada a las Ciencias y las Tecnologías, Universidad de Alicante, San Vicent del Raspeig, \\ 03080 Alicante, Spain \\ 3 Instituto de Astrofísica de Andalucía, CSIC, Apt 3004, 18080 Granada, Spain \\ 4 Florida Space Institute, University of Central Florida, Orlando, FL 32816, USA \\ 5 Instituto de Astrofísica de Canarias (IAC), C/Vía Láctea s/n, 38205 La Laguna, Tenerife, Spain \\ 6 Departamento de Astrofísica, Universidad de La Laguna, 38205 La Laguna, Tenerife, Spain \\ 7 Université Côte d'Azur, Observatoire de la Côte d'Azur, CNRS, Laboratoire Lagrange, Bd. de l'Observatoire, CS 34229, \\ 06304 Nice Cedex 4, France \\ 8 Departmento de Astrofísica, Centro de Astrobiología (CSIC-INTA), ESAC Campus, Camino Bajo del Castillo s/n, Villanueva de \\ la Cañada 28692, Madrid, Spain \\ 9 Spanish Virtual Observatory, Spain \\ 10 Centro de Estudios de Física del Cosmos de Aragón (CEFCA), Unidad Asociada al CSIC, Plaza San Juan 1, 44001 Teruel, Spain \\ 11 Instituto de Astronomia, Geofísica e Ciâncias Atmosféricas, Universidade de São Paulo, 05508-090 São Paulo, Brazil \\ 12 Donostia International Physics Centre (DIPC), Paseo Manuel de Lardizabal 4, 20018 Donostia-San Sebastian, Spain \\ 13 IKERBASQUE, Basque Foundation for Science, 48013 Bilbao, Spain \\ 14 Campus Duque de Caxias, Universidade Federal do Rio de Janeiro, 25265-970 Duque de Caxias, RJ, Brazil
}

Received 22 May 2020 / Accepted 17 May 2021

\section{ABSTRACT}

Context. The Javalambre Photometric Local Universe Survey (J-PLUS) is an observational campaign that aims to obtain photometry in 12 ultraviolet-visible filters $(0.3-1 \mu \mathrm{m})$ over $\sim 8500 \mathrm{deg}^{2}$ of the sky observable from Javalambre (Teruel, Spain). Due to its characteristics and observation strategy, this survey will allow a great number of Solar System small bodies to be analyzed, and with improved spectrophotometric resolution with respect to previous large-area photometric surveys in optical wavelengths.

Aims. The main goal of the present work is to present the first catalog of magnitudes and colors of minor bodies of the Solar System compiled using the first data release (DR1) of the J-PLUS observational campaign: the Moving Objects Observed from Javalambre (MOOJa) catalog.

Methods. Using the compiled photometric data we obtained very-low-resolution reflectance (photo)spectra of the asteroids. We first used a $\sigma$-clipping algorithm in order to remove outliers and clean the data. We then devised a method to select the optimal solar colors in the J-PLUS photometric system. These solar colors were computed using two different approaches: on one hand, we used different spectra of the Sun convolved with the filter transmissions of the J-PLUS system, and on the other, we selected a group of solar-type stars in the J-PLUS DR1 according to their computed stellar parameters. Finally, we used the solar colors to obtain the reflectance spectra of the asteroids.

Results. We present photometric data in the J-PLUS filters for a total of 3122 minor bodies (3666 before outlier removal), and we discuss the main issues with the data, as well as some guidelines to solve them.

Key words. techniques: photometric - catalogs - surveys - minor planets, asteroids: general

\section{Introduction}

The minor bodies that populate the Solar System are considered to contain the most pristine materials within it: remnants

\footnotetext{
* MOOJa catalog of colors and magnitudes are only available at the CDS via anonymous ftp to cdsarc.u-strasbg. fr (130.79.128.5) or via http://cdsarc.u-strasbg.fr/viz-bin/cat/J/A+A/655/ A47
}

of the processes that followed the condensation of the pre-solar nebula and the formation of the planets. Since then, these objects have undergone little or no geological or thermal transformations. However, they have experienced intense collisional events that have affected their shape, size, and surface composition, among other properties; they have also been exposed to different dynamical mechanisms, which have transported these objects from their original locations to their current orbits. Thus, the 
study of minor bodies can provide answers to questions about the origin and evolution of the Solar System.

As pointed out by DeMeo \& Carry (2014), the next step in improving our understanding in this area is to obtain spectra for a large sample of minor bodies. Spectroscopic observations of asteroids provide information about the surface composition of these objects. However, because of the nature of the observational methods used, the number of spectra that are present in the literature is somewhat limited: for visible wavelengths there are spectra available for approximately 3000 objects (e.g., Bus \& Binzel 2002a; Lazzaro et al. 2013; de León et al. 2018), while near-infrared spectra are available for only about 1000 (see DeMeo et al. 2009; Binzel et al. 2019).

Since the beginning of the 21st century, large-area surveys have become a powerful and popular tool with which to answer major questions in the different astronomic fields. Examples of these projects are SDSS (York et al. 2000) and PanSTARRS (Kaiser et al. 2010) at visible wavelengths, and WISE (Wright et al. 2010), AKARI (Ishihara et al. 2010), 2MASS (Skrutskie et al. 2006), VISTA (Sutherland et al. 2015), and UKIDSS (Lawrence et al. 2007) at different infrared wavelengths. Given the vast fractions of the sky covered by these surveys, they provide the opportunity to analyze a large sample of Solar System objects (SSOs) which, although not primary targets, are scattered throughout the obtained data.

Several works have taken advantage of the aforementioned surveys to compile large photometric datasets of minor bodies: Ivezić et al. (2001) extracted data from the SDSS to create the SDSS Moving Object Catalog (MOC), whose last version contains data for more than 450000 objects ${ }^{1}$, Popescu et al. (2016), using data from the VISTA Hemisphere Survey (VHS), created the Moving Objects from VISTA Survey (MOVIS) catalog with data for more than 50000 objects, and Mainzer et al. (2011) obtained mid-infrared photometry for more than 150000 objects from the WISE survey, and produced the largest database (so far) of sizes and albedos of asteroids. These datasets all have a limited number of filters (five in the SDSS and four in VISTA and WISE). However, even with only four or five filters, the catalogs are extensive, because of the large number of observed objects. Some of the most relevant works derived from each of the previously mentioned surveys are Ivezić et al. (2002), Parker et al. (2008), Carvano et al. (2010), Hasselmann et al. (2011, 2015), Masiero et al. (2011, 2013, 2014), Rivkin (2012), Alí-Lagoa et al. (2013), Mainzer et al. (2014), Licandro et al. (2017), Popescu et al. (2018), Morate et al. (2018).

\section{The Javalambre Photometric Local Universe Survey}

J-PLUS is a photometric sky survey designed to cover $\sim 8500 \mathrm{deg}^{2}$ of the sky and to observe and characterize galaxies and stars of the Milky Way halo in order to provide data with a wide range of astrophysical applications (Cenarro et al. 2019) ${ }^{2}$.

J-PLUS observations are performed with the JAST/T80 0.8-m telescope located in the Observatorio Astronómico de Javalambre (OAJ, Teruel, Spain; Cenarro et al. 2014) using the panoramic camera T80Cam (Marin-Franch et al. 2015), which has a $2 \mathrm{deg}^{2}$ field of view (FoV), covering a wavelength region between 0.35 and $1 \mu \mathrm{m}$ by means of a 12 filter system: five intermediate-band filters (four of them equivalent to the SDSS $g^{\prime}, r^{\prime}, i^{\prime}$, and $z^{\prime}$ filters, the other one being a modified version of the SDSS $u^{\prime}$ filter), and seven narrow-band filters designed

\footnotetext{
1 An updated version of the MOC catalogue can be found at http: //svo2.cab.inta-csic.es/vocats/svomoc

2 See also https://www.j-plus.es/
}

specifically for J-PLUS (see Fig. 1). The observation strategy is to obtain three exposures per filter in sequence, with the total time of acquisition in the 12 filters for the same field being approximately one hour. Taking into account (a) the large fraction of sky coverage, (b) the spectral resolution, better than previous visible large-area photometric surveys, and (c) the observational strategy, we expect to obtain improved spectrophotometry (compared to existing datasets) for a relatively large number of minor bodies in the Solar System.

Being such a large survey, the photometric calibration of J-PLUS data follows a complex procedure. The data presented here rely on a calibration that uses the locus of the main sequence stars observed in the footprint of the survey, anchoring the colors to Pan-STARSS, as described in detail by López-Sanjuan et al. (2019).

Another interesting feature of the J-PLUS survey is its powerful photometric resolution in the UV region: five filters cover the $0.3-0.45 \mu \mathrm{m}$ range. The other prominent survey that offers UV spectral information for minor bodies (for a total of 589 objects) is the Eight Color Asteroid Survey (ECAS, Zellner et al. 1985), which has only three filters in the same region: $s, u$, and $b$, centered at $0.337,0.359$, and $0.437 \mu \mathrm{m}$ respectively (see Fig. 2). We note that, although the central wavelength of the $b$ ECAS filter is $0.437 \mu \mathrm{m}$, this filter covers a broad wavelength range, that is, it includes nonUV information, while all the J-PLUS UV filters are narrow-band (except for the $u$ filter), giving specific coverage for this region. As we show in the following section, the number of Solar System objects that we expect to recover from J-PLUS observations in this spectral region is approximately one order of magnitude higher than the number of available objects in the literature, which will improve our current knowledge of minor bodies in a spectral region that has not yet been deeply explored.

The present work is organized as follows: in Sect. 2 we give a brief summary of the survey status, we present the catalog and its most remarkable properties, and we also discuss the removal of outliers. In Sect. 3 we present a method to select the optimal solar colors in the J-PLUS photometric system, needed to later compute the reflectance photospectra. In Sect. 4 we discuss the data quality and the main issues that need to be addressed. Finally, in Sect. 5 we summarize the contents of the paper, proposing possible future analyses, as well as ways to improve the data extraction from the survey observations.

Finally, we want to stress that the scope of the present work is to select photometric data from minor bodies contained in the first data release (DR1) of J-PLUS, providing a large sample of the colors of minor bodies - which can be used to compute very-low-resolution spectra (or photospectra) - rather than performing in-depth analyses of the obtained data. The present work is one of a list of articles presenting the diverse aspects of J-PLUS DR1 (e.g., Logroño-García et al. 2019; Whitten et al. 2019; Solano et al. 2019).

\section{Observations and the MOOJa (Moving Objects Observed from Javalambre) catalog}

Solar System objects move over the sky background. This means that, depending on the exposure time of the image, the proper motions of the object, and the pixel scale of the detector, a particular object might appear as a trace instead of a point source, making it difficult or even impossible to compute its photometry. Fortunately, the majority of SSOs have proper motions smaller than $1^{\prime \prime} \min ^{-1}$ (mainly objects located in the Main Belt and beyond), which, combined with the mean exposure times in 


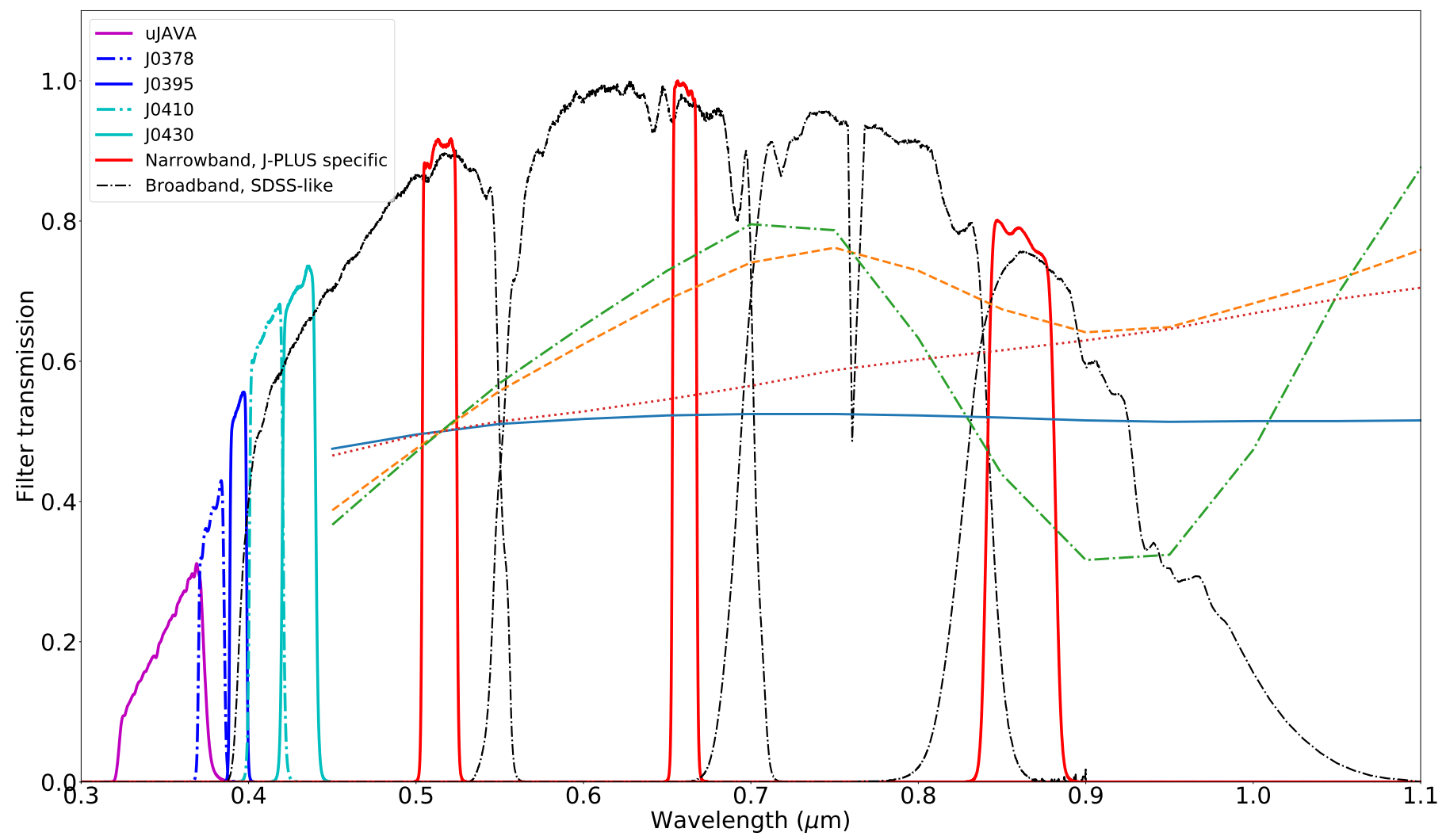

Fig. 1. J-PLUS filter system. The solid magenta line represents the $u$ JAVA filter; the blue adjacent curves (dotted and solid) represent the $J 0378$ and $J 0395$ filters, respectively; the following cyan curves (dotted and solid) represent the $J 0410$ and $J 0430$ filters; the three solid red lines are the narrow-band filters $J 0515, J 0660$, and $J 0861$. These eight filters were designed specifically for J-PLUS. The remaining dashed black lines are the broad-band SDSS filters: from left to right, lines: $g, r, i, z$. For comparison, the spectral templates of four taxonomical classes (source: http://smass .mit. edu/busdemeoclass.html) are included: C (solid, blue), X (dotted, red), S (dashed, orange), and V (dash-dotted, green). The spectra have been shifted for better graphical representation, and normalized to 0.515 microns, which, of the central wavelengths of every filter, is the nearest value to 0.55 microns, the most commonly used value for normalization of reflectance spectra of asteroids in the visible wavelength range. We note that the spectral templates do not cover the ultraviolet (UV) region (below 0.45 microns).

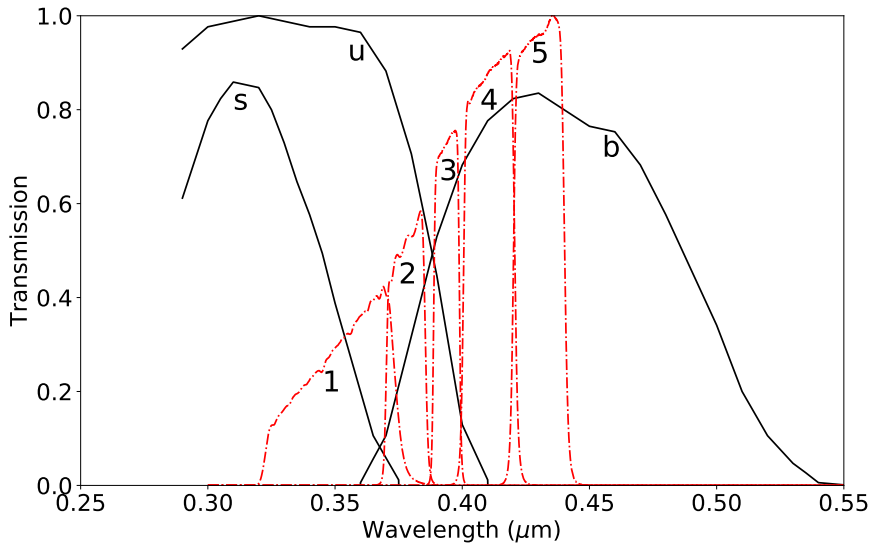

Fig. 2. Graphical comparison of the transmissions of the ECAS filters (black solid lines), and the J-PLUS filters (red dashdotted lines) in the UV region. The numbers correspond to: 1-u, 2-J0378, 3-J3095, 4-J0410, 5-J0430. ECAS transmissions are normalized to unity at the maximum of the $u$ filter, and J-PLUS transmissions are normalized to unity at the maximum of the $J 0430$ filter.

every filter (as we discuss in the following paragraph), is sufficient for most of the asteroids observed by the survey to be detected as point sources, allowing computation of their photometry without issues.
Although the scientific aim of J-PLUS was not specifically to observe minor bodies, its observational strategy allows their detection within the survey images. The observations follow a sky tiling: each tile has the size of the T80Cam FoV, and in order to guarantee coverage continuity, neighboring pointings overlap in the FoV limits. For each pointing of the survey, all 12 filters are observed consecutively, following a predefined sequence. Every J-PLUS pointing is made of 36 exposures ( 3 for each filter), and the 3 exposures in a given filter are consecutive, with a small dithering of $10^{\prime \prime}$ between them that allows the removal of bad pixels or columns. The three exposures are then combined, resulting in 12 images per field (one per filter). The mean exposure time per image in the broad-band filters is $\sim 1 \mathrm{~min}$, and in the case of the narrow-band filters, is $\sim 2-3 \mathrm{~min}$. The complete filter sequence is the following: $i, u, J 0430, J 0861, J 0515, J 0410, r$, $J 0378, J 0660, g, J 0395$, and $z$. This filter acquisition order has been defined in a way that observations between filters with similar central wavelengths are performed within long enough time intervals for nonsidereal movement detection and simultaneous minimization of long filter wheel rotations.

\section{The MOOJa catalog}

The J-PLUS DR $1^{3}$ contains images of 511 fields collected from November 2015 to January 2018. Its sky coverage is

3 http://archive.cefca.es/catalogues/jplus-dr1/ 


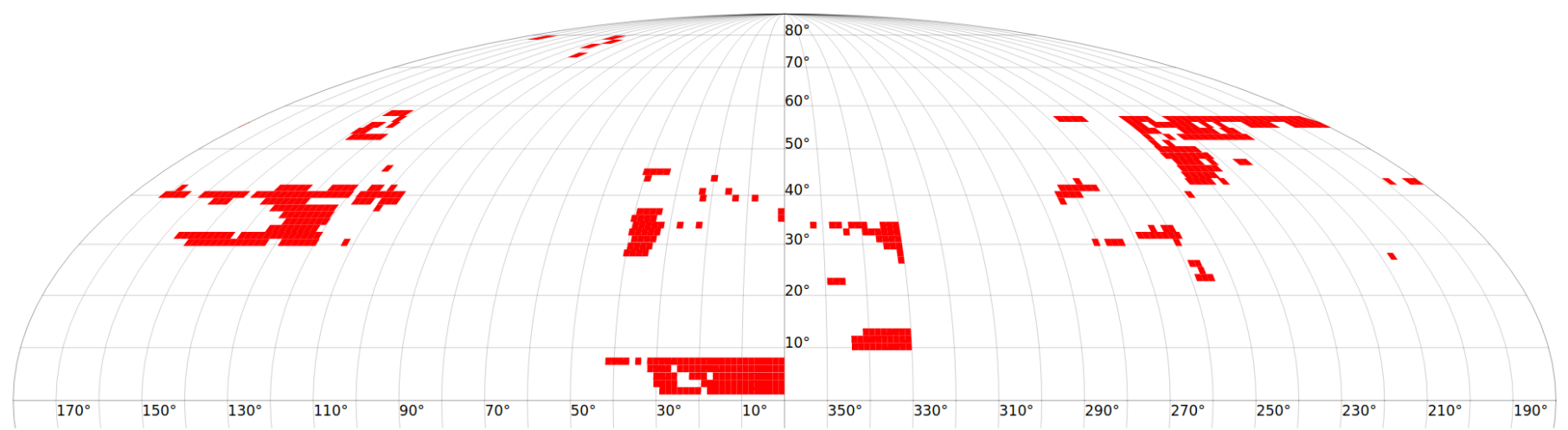

Fig. 3. J-PLUS DR1 skymap coverage, represented using the ICRS. Source: J-PLUS website (http://archive.cefca.es/catalogues/ jplus-dr1/coverage_map.html).

shown in Fig. 3, using the International Celestial Reference System (ICRS). The total nonoverlapped area is $\sim 900 \mathrm{deg}^{2}$, that is, approximately $10 \%$ of the intended area has already been observed. This is the dataset from which the minor body data have been extracted.

The asteroids present in the J-PLUS DR1 are recovered using the SSOS pipeline, a versatile piece of software developed to detect both known and unknown SSOs in astronomical images ${ }^{4}$. A detailed description of the pipeline setup is given in Mahlke et al. (2019). SSOS is applied to each J-PLUS field individually. We briefly summarize the pipeline in the following steps: first, all sources present in the 12 images of each field are cataloged using SExtractor (Bertin \& Arnouts 1996). Next, the sources detected in the individual images are associated to their corresponding observation epochs using SCAMP (Bertin 2006). Subsequently, SSOs are separated from the stars, galaxies, and other sources within the sample primarily by evaluating their apparent motions. Finally, using the SkyBoT service (Berthier et al. 2006), the output sample is cross-matched with the known population of asteroids. The astrometry of both known and unknown objects is reported to the Minor Planet Centre (MPC). However, the positions of the unknown objects are not sufficient to determine their orbits. Positions are combined with other isolated tracklets by the MPC, hopefully producing orbits of new asteroids. Thus, the unknown objects have been left out of the catalog, because none of them have been confirmed by the MPC as a new discovery. Magnitudes were computed using SExtractor by fitting adaptive apertures to the pixels of the sources in the images. SExtractor automatically computes instrumental magnitudes (calibrated in the $\mathrm{AB}$ system) from the pixel values inside the aperture by relying on telescope and image metadata present in the FITS headers of the files.

The majority of the asteroids in the catalog were observed in a single pointing during a complete filter sequence, or run. However, some of these objects were not detected in all of the filters, probably because of their brightness. In addition, there were some cases in which the asteroid was observed in two consecutive runs during the same night (in which cases the run had to be restarted), or even in different nights. Whenever an asteroid was detected in more than one run in the same night, we divided the data into blocks in which the elapsed time from the first to the last filter acquisition is $60 \mathrm{~min}^{5}$ at most. We then selected the block with the maximum number of different filters, and, when there was more than one block that fulfilled this condition, we

\footnotetext{
4 https://ssos.readthedocs.io/

5 See Sect. 4 for details on the 60 min limit.
}

selected the block corresponding to the data with the smallest mean errors. If an asteroid was observed in different nights, but in only one run each night, we selected the data corresponding to the night with the maximum number of different filters, and again, if this number was the same for the different nights, we selected the data with the smallest errors.

The full catalog presents data for a total of 3666 objects: 3570 numbered asteroids, plus 96 asteroids with only a provisional designation. Of these, 359 were observed in all 12 filters; 386 in at least the 5 UV filters; and 2476 in at least the 7 nonUV filters. In Fig. 4, we can see the distribution of the observed objects throughout the main asteroid belt.

To remove outliers, we applied a $\sigma$-clipping algorithm in the color space. We do not expect the color distributions to be completely Gaussian-like; however, despite this limitation, we proceed assuming that they are, because this assumption does not appear to introduce unwanted features in the data-rejection process. We computed colors $m_{f}-m_{J 0515}{ }^{6}$, and then computed the mean and standard deviation of the corresponding distributions. We selected data satisfying $|x-\bar{x}|>2.5 \sigma^{7}$, where $x$ is the color and $\sigma$ is the standard deviation of the sample, discarding the $m_{f}$ observation of the corresponding color. The process was iterated until no outliers were found. The final color distributions are shown in Fig. 5. A total of 460 objects were removed by the $\sigma$-clipping procedure. In Fig. 6, we show how many objects have data colors for each filter (referred to J0515), before and after the outlier removal.

We must bear two things in mind: first, all the objects that do not have observations in the $J 0515$ filter were removed because we cannot use this filter to normalize the corresponding photospectrum; second, and related to the previous point, if the observation in the J0515 filter is considered an outlier, that object will also be removed because, again, even if the other observations are valid, the photospectrum cannot be constructed because of the lack of reliability of the reference observation. For this reason, another 84 entries were removed from the original dataset. Therefore, we provide two catalogs: one with all the recovered data in case the reader wants to perform their own analysis, and another that we consider a sufficiently robust dataset, with colors referring to the normalization filter $\left(m_{f}-m_{J 0515}\right)$, ready for use in computing the corresponding photospectra (see Sect. 3 ).

\footnotetext{
6 We chose $m_{J 0515}$ as the normalization filter, see below.

7 This threshold was established based on a compromise between data completeness and cleanness: more restrictive thresholds removed observations that were not really flawed, while less restrictive thresholds did not allow the removal of data that were obvious outliers.
} 

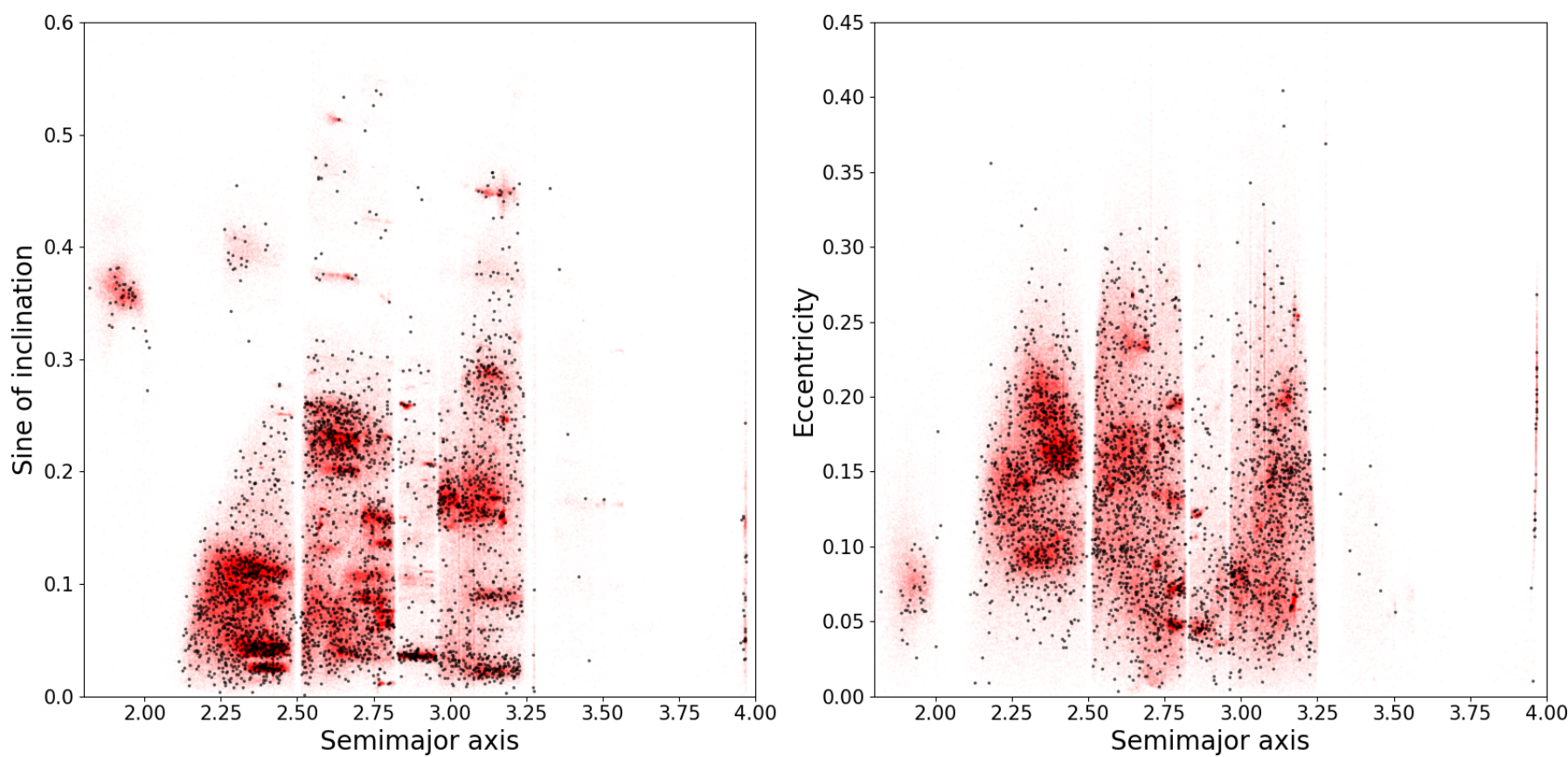

Fig. 4. Asteroids within the MOOJa catalog for which proper elements are available (black) superimposed on all proper elements for minor bodies in the Solar System (red). We show their semimajor axis values vs. the sine of inclination of the orbit (left panel) and vs. eccentricity (right panel). Data available at the AstDyS-2 website (https://newton. spacedys.com/ astdys2/propsynth/all. syn).
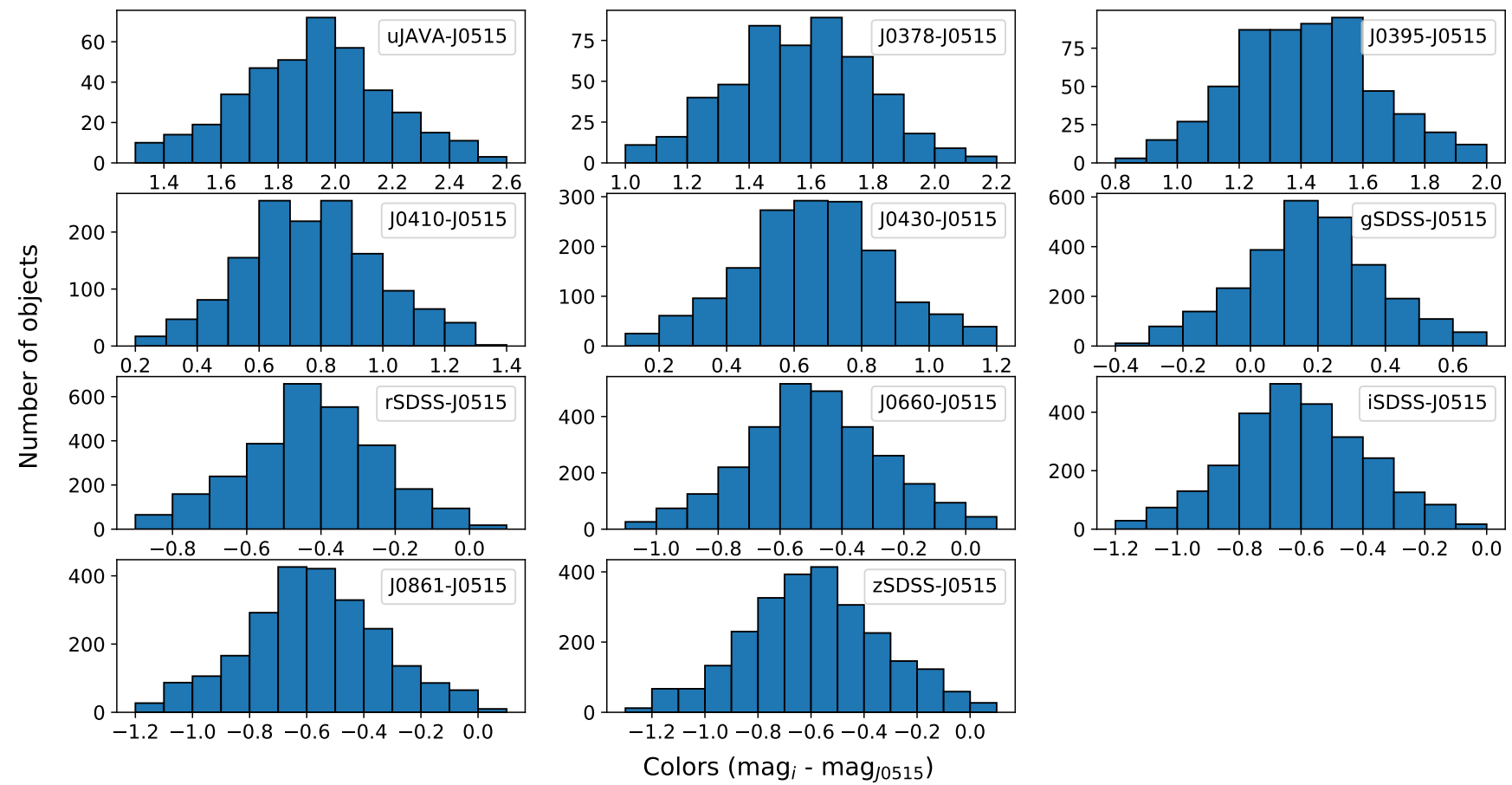

Fig. 5. Histograms for the color distribution after sigma clipping to remove outliers. As mentioned in the main text, all the colors refer to $m_{J 0515}$, which is the filter that we used for normalization. The bin size is $0.10 \mathrm{mag}$.

The provided color catalog consists of data for 3122 asteroids. Appendix A presents some color-color distributions as a brief proof-of-concept for the reader (as the main focus of this work is to present photospectra rather than color analyses) and to show that the data presented in the MOOJa catalog are sufficient to make an apparent differentiation between the $\mathrm{C}$ and $\mathrm{S}$ complexes. Out of those 3122 objects, 278 were observed in every filter, and therefore we will be able to construct their complete photospectra. In addition, another 2005 objects have data for at least six colors (i.e., observed in seven filters, including the normalization filter, J0515). Figure 7 shows the number of objects for which data are available for a given total number of colors.

The limiting magnitude of the survey can be inferred by plotting the photometric errors versus the predicted magnitude for the detected objects (see Fig. 8). The limiting magnitude depends on the filter: photometric data with magnitude errors smaller than 


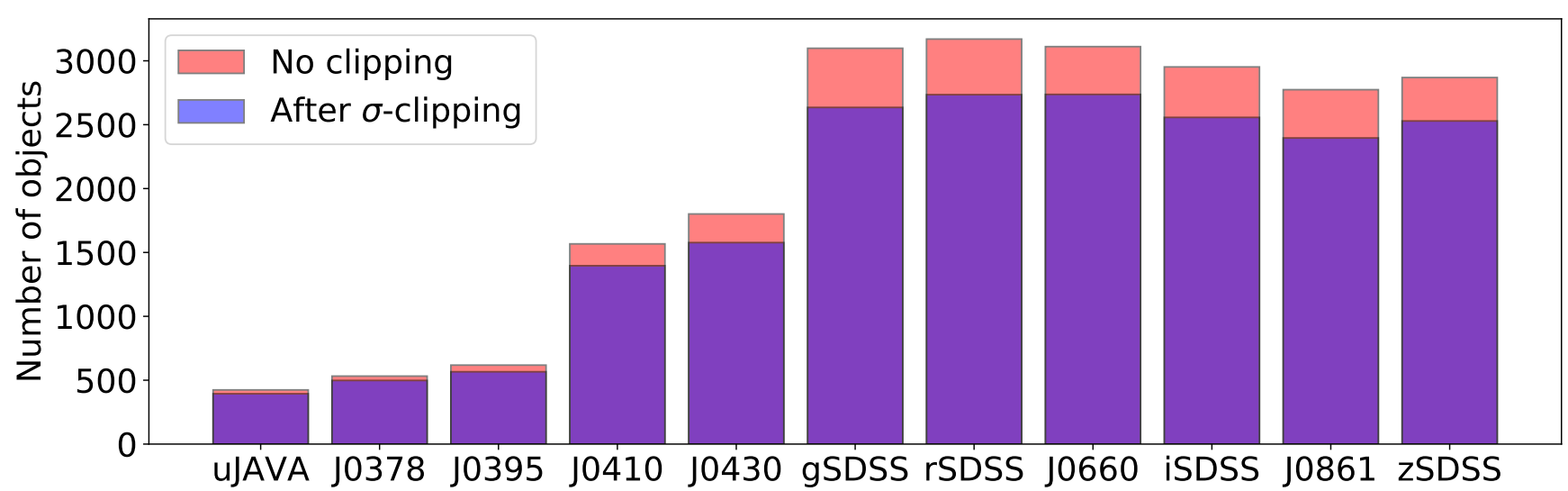

Fig. 6. Number of objects observed in a specific filter and in $J 0515$.

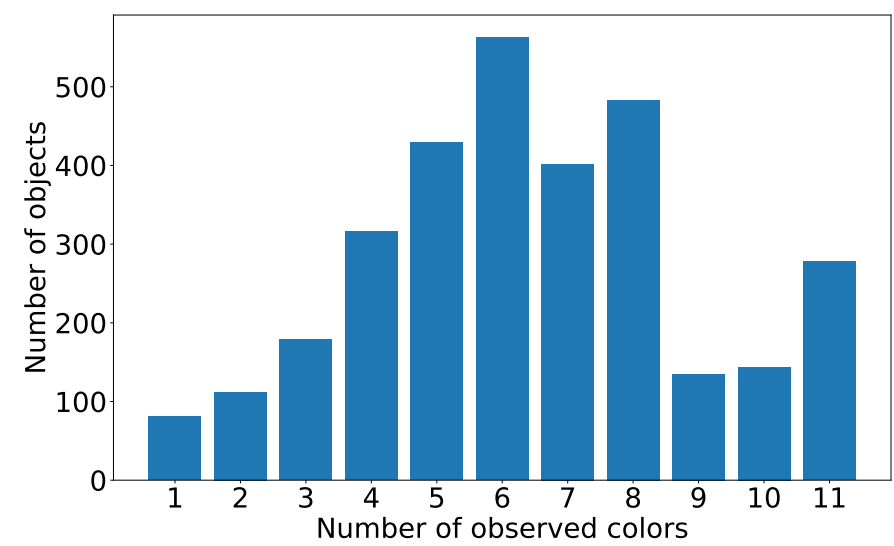

Fig. 7. Number of objects versus number of colors remaining after sigma-clipping.

0.1 can be obtained for the $u$ JAVA, J0378, and $J 0395$ filters (UV region) for $V$ magnitudes brighter than $V \sim 17.5$; for $J 0410$ and $J 0430$ (transition region from the UV to the visible) this limit goes up to $V \sim 19$. Finally, for the visible filters ( $g$ SDSS, J0515, $r$ SDSS, J0660, iSDSS, J0861, and zSDSS) the limiting magnitude is $V \sim 20.5$. This explains why there are data for smaller objects in the redder filters, as opposed to the bluer filters where only sufficiently large objects were detected in the UV region (see Fig. 9).

Taking into account the area that the DR1 has already covered, a conservative estimate of the total number of minor bodies that will be recovered after the survey is completed is approximately 18000 objects, of which we expect about 1800 to be observed in all 12 filters, about 2000 in at least the 5 UV filters, and about 12000 in at least the 7 nonUV filters.

The full catalog consists of a list of asteroids labeled by their identification number or provisional designation (first column of the catalog). After the ID number, there are three columns regarding each of the 12 filters: the first column is the detected magnitude, the second is the error in the magnitude measurement, and the third is the MJD in the middle of the three exposures in the corresponding filter. For each asteroid, we also provide (when available) their corresponding taxonomic classification according to Carvano et al. (2010), computed using SDSS data, in the second-to-last column of the catalog. The absolute magnitudes, $H$ (from the JPL website), for each object are given in the penultimate column. Finally, in the last column, and for quick reference, we show the number of filters in which the object has been observed.
The color catalog presents the colors as $m_{f}-m_{J 0515}$. The catalog also has the identification number of each entry in the first column. It also provides four entries per color, with the color value, its corresponding error, the elapsed time between the observation in both filters, and the MJD of the observation in the $m_{f}$ filter. As was the case for the full cata$\log$, the columns with taxonomy and absolute magnitude are included. In this case, we also provide the predicted $V$ magnitude at the moment of the observation (extracted using the JPL Horizons service) in the penultimate column. The last column shows the number of colors for which data are available for each object (analogous to the last column of the full catalog). Both datasets can be downloaded from the CDS repository.

\section{The solar colors in the J-PLUS photometric system}

Solar System objects reflect the light of the Sun at the wavelengths observed by J-PLUS. It is therefore necessary to remove this signature to obtain reflectance values. These reflectance values, the spectra of minor bodies, are used to infer their surface composition by comparison with meteoritic samples.

In order to transform J-PLUS magnitudes into reflectances, we followed a simple equation, where the reflectance value for each filter, $R_{\mathrm{f}}$, is computed as

$R_{\mathrm{f}}=10^{-0.4\left(C_{\mathrm{f}, \mathrm{ast}}-C_{\mathrm{f}, \odot}\right)}$

where $C_{\mathrm{f}}=m_{f}-m_{J 0515}$ is the color, or magnitude difference, between filter $f$ and the normalization filter $(J 0515)$, for both the asteroid and the Sun at the nominal central wavelengths for each filter (see Table 1).

From Eq. (1) it is clear that solar colors $\left(C_{\mathrm{f}, \odot}\right)$ in the pertinent filters are needed to obtain reflectances. Values of $C_{\mathrm{f}, \odot}$ have not yet been determined by the collaboration, at least not for all filters involved. Therefore, in order to obtain reliable $C_{\mathrm{f}, \odot}$ we used nine sets of data, following two different approaches: one theoretical approach (four sets) based on the published spectra of the Sun, and one empirical approach (five sets) based on J-PLUS colors of solar type stars.

In the following sections we provide a more detailed description of the solar spectra that we used, as well as of the solar analog selection process. In the last section, we discuss which of these spectra would produce the best solar colors. 
D. Morate et al.: J-PLUS: A first glimpse at the spectrophotometry of asteroids. The MOOJa catalog

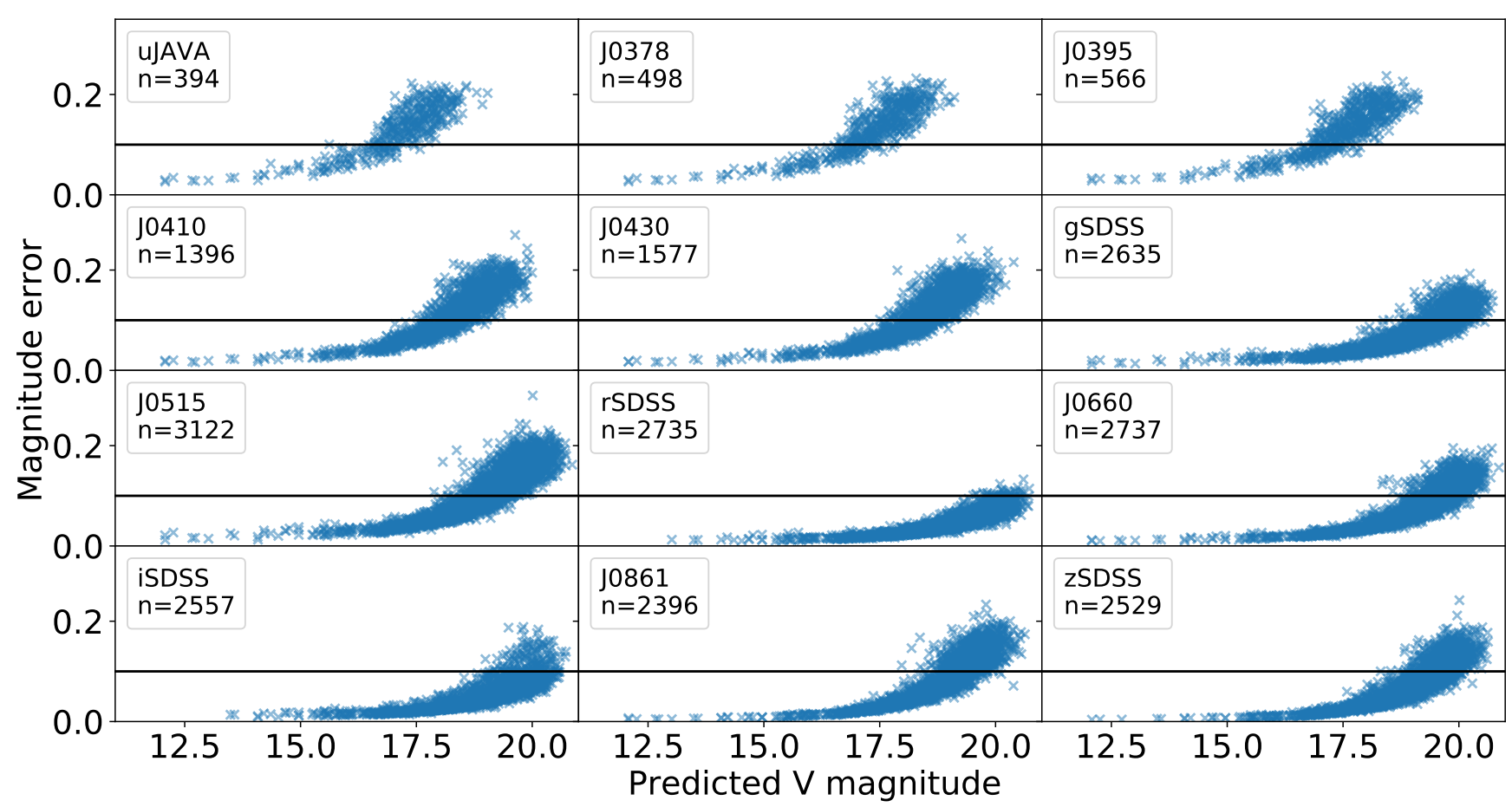

Fig. 8. Distribution of the errors associated to each observation (for the sigma-clipped dataset), relative to the predicted apparent $V$ magnitude for the 12 filters of J-PLUS. The horizontal black line corresponds to an error of $0.1 \mathrm{mag}$.

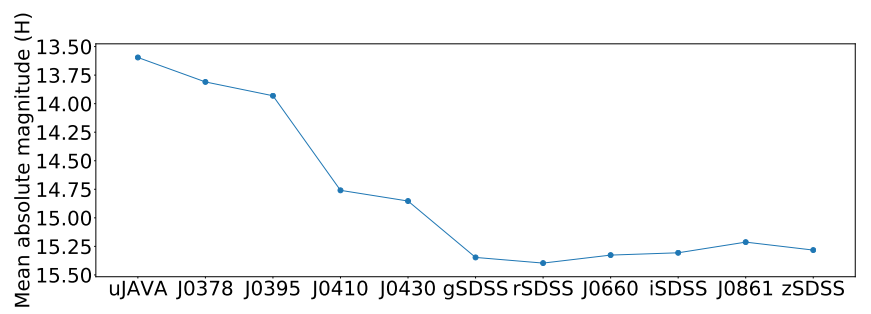

Fig. 9. Mean absolute magnitude per color. Magnitudes extracted from the JPL website.

Table 1. Central wavelengths and bandwidths of the J-PLUS filters.

\begin{tabular}{llcc}
\hline \hline ID & $\begin{array}{l}\text { Filter } \\
\text { name }\end{array}$ & $\begin{array}{c}\text { Central } \lambda \\
(\mu \mathrm{m})\end{array}$ & $\begin{array}{c}\text { Bandwidth } \\
(\mu \mathrm{m})\end{array}$ \\
\hline 1 & $u$ & 0.3485 & 0.0508 \\
2 & $J 0378$ & 0.3785 & 0.0168 \\
3 & $J 0395$ & 0.3950 & 0.0100 \\
4 & $J 0410$ & 0.4100 & 0.0200 \\
5 & $J 0430$ & 0.4300 & 0.0200 \\
6 & $g$ & 0.4803 & 0.1409 \\
7 & $J 0515$ & 0.5150 & 0.0200 \\
8 & $r$ & 0.6254 & 0.1388 \\
9 & $J 0660$ & 0.6600 & 0.0145 \\
10 & $i$ & 0.7668 & 0.1535 \\
11 & $J 0861$ & 0.8610 & 0.0400 \\
12 & $z$ & 0.9114 & 0.1409 \\
\hline
\end{tabular}

Notes. Source: http://www.j-plus.es/survey/instrumenta tion

\subsection{Theoretical solar colors}

There exist several solar spectra in the literature that have been and are used by the community. These are all extraterrestrial solar irradiance spectra obtained at zero airmass and are all based on data from satellites, space shuttle missions, high-altitude aircraft, rocket soundings, ground-based solar telescopes, and (or) modeled spectral irradiance. Out of all the available spectra, we arbitrarily chose four of them in order to have a sufficient number of different sources and results. The ones used for this analysis were:

- 1985 Wehrli Standard Extraterrestrial Solar Irradiance Spectrum.

- 2000 ASTM Standard Extraterrestrial Spectrum Reference E-490-00.

- MODTRAN ETR Thuillier Spectrum.

- PMOD/WRC Solar Reference Spectrum.

Further references can be found in Wehrli (1985), Fröhlich \& Lean (1997), Thuillier et al. (2003, 2004), Chance \& Kurucz (2010), and Haberreiter et al. (2017). All four spectra are available online ${ }^{8}$. To compute $C_{\mathrm{f}, \odot}$, we convolved the four spectra with the J-PLUS filters transmission. The detector efficiency and atmospheric transmission information are already included in the filter transmission curves. The computed solar colors are shown in Table 2.

\subsection{Empirical solar colors}

The second approach used to solve the problem of obtaining the solar colors in the J-PLUS photometric system was to analyze the DR1, searching for a set of possible Sun-like stars observed in the 12 filters. Solar-type stars, solar analogs, and solar twins are stars that are particularly similar to the Sun, the solar twin being most like the Sun followed by solar analogs and then solartypes (Soderblom \& King 1998). Solar twins should be indistinguishable from the Sun, this means:

8 The first three spectra are available at https://rredc.nrel.gov/ solar/spectra/, and the PMOD spectrum is available at the public FTP of the PMOD/WRC (ftp://ftp.pmodwrc.ch/pub/data/ SolarReferenceSpectrum/). 
Table 2. Solar colors for each J-PLUS filter, computed from solar spectra (theoretical) and from Sun-like stars observed in J-PLUS in all 12 filters (empirical).

\begin{tabular}{|c|c|c|c|c|c|c|c|c|c|c|c|}
\hline $\begin{array}{l}\text { Spectrum/ } \\
\text { Dataset }\end{array}$ & $u$ & $J 0378$ & $J 0395$ & $J 0410$ & $J 0430$ & $g$ & $r$ & $J 0660$ & $i$ & $J 0861$ & $z$ \\
\hline \multicolumn{12}{|l|}{ Theoretical } \\
\hline 1985 Wehrli & 1.4913 & 1.2941 & 1.1518 & 0.5876 & 0.5118 & 0.1757 & -0.2877 & -0.3269 & -0.3979 & -0.4180 & -0.4202 \\
\hline 2000 ASTM & 1.5110 & 1.3023 & 1.1455 & 0.5853 & 0.5097 & 0.1750 & -0.2879 & -0.3275 & -0.3985 & -0.4173 & -0.4338 \\
\hline Thuillier & 1.4325 & 1.2426 & 1.1240 & 0.5561 & 0.4993 & 0.1614 & -0.2777 & -0.3157 & -0.4048 & -0.4387 & -0.4251 \\
\hline PMOD/WRC & 1.4749 & 1.2665 & 1.1499 & 0.5707 & 0.4913 & 0.1605 & -0.2874 & -0.3156 & -0.4072 & -0.4154 & -0.4245 \\
\hline \multicolumn{12}{|l|}{ Empirical } \\
\hline G1 & 1.4048 & 1.1210 & 1.0886 & 0.5541 & 0.5015 & 0.1456 & -0.3288 & -0.3752 & -0.4997 & -0.5330 & -0.5587 \\
\hline $\mathrm{G} 2$ & 1.4042 & 1.1195 & 1.0883 & 0.5537 & 0.5013 & 0.1455 & -0.3288 & -0.3753 & -0.4994 & -0.5328 & -0.5585 \\
\hline G3 & 1.4828 & 1.2037 & 1.1375 & 0.5778 & 0.5125 & 0.1489 & -0.3265 & -0.3742 & -0.4903 & -0.5169 & -0.5443 \\
\hline G4 & 1.4808 & 1.2014 & 1.1361 & 0.5768 & 0.5125 & 0.1483 & -0.3261 & -0.3746 & -0.4901 & -0.5169 & -0.5444 \\
\hline G5 & 1.5256 & 1.2784 & 1.1909 & 0.5853 & 0.5123 & 0.1579 & -0.3296 & -0.3728 & -0.4923 & -0.5167 & -0.5331 \\
\hline
\end{tabular}

Notes. All the colors refer to the $J 0515$ filter, the normalization filter that we used for J-PLUS spectrophotometric data (i.e., $m_{i}-m_{J 0515}$ ).

1. Stellar parameters similar to those of the Sun.

2. Age within $\sim 1 \mathrm{Gyr}$, so that the evolutionary state is comparable.

3. No known stellar companion, because the Sun has none.

We stress here that this type of star is not particularly targeted by the survey strategy and we have to rely on serendipitous observations. The most commonly used stellar parameters are effective temperature, gravity, and metallicity (represented as $T_{\text {eff }}, \log g$, and $[\mathrm{Fe} / \mathrm{H}]$, respectively). In the case of the Sun, these parameters are $T_{\text {eff }}=5777 \pm 10 \mathrm{~K}, \log g=4.4374 \pm 0.0005$, and zero metallicity ${ }^{9},[\mathrm{Fe} / \mathrm{H}]=0$ (see Smalley 2005 and references within).

Stellar parameters can be computed from J-PLUS data. To this end, we used a supervised machine learning model discussed by Galarza et al. (in prep.) based on the random forest algorithm (see Breiman 2001 and references within).

The model needs a subset of well-known data in order to be trained: this training subset was obtained by cross-checking the J-PLUS DR 1 catalog with the SEGUE survey from the SDSS. It uses a set of multiple J-PLUS colors for a given object as input parameters, and then returns estimated values of effective temperature, surface gravity, and metallicity as output parameters.

Using this method, we managed to select a list of 8633 stars within J-PLUS, with $5767 \mathrm{~K}<T_{\text {eff }}<5787 \mathrm{~K}$, thus meeting the first criterion: we refer to this dataset as the G1 set. The remaining criteria, however, were not fully met because of the low spectral resolution and the need for a thorough analysis of the data in the G1 set, which is out of the scope of the present work. To compensate for this, we selected several subsets of the G1 set, producing a total of five sets of Sun-like stars, which we named G1-G5. These subsets were arbitrarily selected, and filtered using the following criteria (the number of objects in each group is shown in parentheses):

- G1: Stars with $5767 \mathrm{~K}<T_{\text {eff }}<5787 \mathrm{~K}(8633)$.

- G2: Stars with $5772 \mathrm{~K}<T_{\text {eff }}<5782 \mathrm{~K}$ (4792).

- G3: Stars with $5767 \mathrm{~K}<T_{\text {eff }}<5787 \mathrm{~K}$ and photometric errors in every filter of less than $0.01 \mathrm{mag}(691)$.

- G4: Stars with $5772 \mathrm{~K}<T_{\text {eff }}<5782 \mathrm{~K}$ and photometric errors in every filter of less than $0.01 \mathrm{mag}$ (409).

- G5: Stars with $5767 \mathrm{~K}<T_{\text {eff }}<5787 \mathrm{~K},-0.3<[\mathrm{Fe} / \mathrm{H}]<$ 0.3 and $4.1<\log g<4.7$ (71).

In Table 2 we can see $C_{\mathrm{f}, \odot}$ derived for the G1 to G5 datasets.

9 The metallicity of a star is defined relative to the Sun.

\subsection{The optimal solar colors for MOOJa photospectra}

In order to check the quality of the determined solar colors and to select the dataset that best reproduces the asteroid reflectances, we used a sample of known spectra of minor bodies, and then compared it with the reflectances that we retrieved from the MOOJa catalog using each set of solar colors.

The spectra of minor bodies were taken from the several dedicated surveys that have observed, to date, more than 3000 spectra in the visible spectral range. The most prominent ones, that is, those with the greatest number of observed objects and the best spectral resolution, are SMASS (I and II, Xu et al. 1995; Bus \& Binzel 2002b), S3OS2 (Lazzaro et al. 2004), and PRIMASS (de Leon et al. 2018). We used these, together with other well-known datasets (ECAS, Zellner et al. 1985; Sawyer, Sawyer 2005; 24 Color-Asteroid-Survey, Chapman et al. 2005), for comparison with the J-PLUS spectrophotometry.

For each object present in the literature and in J-PLUS DR1 we first convolved their spectra with the J-PLUS transmission filters to obtain the reflectances in each fitler using

$R_{\mathrm{f}}=\frac{\sum_{\lambda} T_{\mathrm{f}} S \lambda \mathrm{d} \lambda}{\sum_{\lambda} T_{\mathrm{f}} \lambda \mathrm{d} \lambda}$,

where $T_{\mathrm{f}}$ is the filter transmission, $S$ is the spectrum reflectance, and $\lambda$ and $\mathrm{d} \lambda$ are the wavelength of the spectrum and its step, respectively. This means that, to convolve the spectra with the filters, we needed to interpolate the filter transmissions at the corresponding spectral wavelengths in order to have one point of the filter transmission curve for each reflectance and wavelength value.

In the cases where the error information associated to each point of the literature spectra was available, the errors were propagated according to uncertainty propagation theory (this is the case for SMASSII, S3OS2, ECAS, and 24CAS). When the spectra did not have error information available (SMASSI, Sawyer, and PRIMASS), the errors were computed as the absolute value of the difference, point to point, between the spectrum and its fourth-order polynomial fit. The corresponding photospectra, together with the J-PLUS observations, are shown in Appendix B.

There are a number of factors that may account for differences between spectra observed by different surveys, ranging 
from actual spectral variation in the asteroids to systematic factors in the surveys and the choice of solar analog stars. We therefore decided to parameterize this difference, taking into account the differences in reflectance, as well as the errors associated to both spectrum and photospectrum. This parameter, $Q$, roughly quantifies how similar one literature spectrum is to its corresponding J-PLUS photospectrum, and its definition is:

$\delta Q_{i}=\sqrt{\frac{\left(R_{i, \text { Lit. }}-R_{i, \text { JPLUS }}\right)^{2}}{\sigma_{i, \text { Lit. }}^{2}+\sigma_{i, \text { JPLUS }}^{2}}}, \quad Q=\frac{\sum_{i=1}^{N} \delta Q_{i}}{N-1}$,

where $R_{i, \text { Lit. }}$ is the reflectance value for the filter $i$ obtained after the convolution of the literature spectrum with the J-PLUS filters; $R_{i \text { JPLUS }}$ is the reflectance value for the filter $i$ computed from the J-PLUS magnitudes after removing the solar colors; $\sigma_{i, \text { Lit. }}$ and $\sigma_{i, \text { JPLUS }}$ are their respective errors; and finally, $N$ is the number of filters in which the asteroid has been observed in J-PLUS.

Each J-PLUS photospectrum has as many associated $Q$ indexes as available spectra in the literature (see Table 3 for information of the analyzed photospectra). Also, for each solar color set, we therefore have a $Q$ distribution. In accordance with the definition of the $Q$ index, we expect that, the closer the value of $Q$ is to zero, the more similar a J-PLUS spectrum will be to its literature counterparts. Thus, we consider the distribution with the smallest mean value of $Q$ as the best approximation, and we select the solar colors that produced that distribution as the best suited to compute the photospectra of the asteroids in the MOOJa catalog. The analysis of the resulting distributions is discussed in the following paragraphs.

The solar colors shown in Table 2 were used to construct the photospectra of the asteroids in Table 3. Then, after comparing these with the literature spectra, we obtained the $Q$ distribution associated to the use of each solar color set. In Fig. 10 we show the cumulative distribution function $(\mathrm{CDF})$ for every $C_{\mathrm{f}, \odot}$ set derived from a different solar spectrum. The mean values of $Q$ for the four distributions are shown in Table 4 . We find that the CDF with the minimum mean value of $Q$ is the one associated to the 2000 ASTM E-490 spectrum; thus, we selected the corresponding solar colors as the best choice derived from the theoretical approach.

In the case of the empirical colors, we note that the colors produced by sets G1 and G2 are very similar, as are those related to G3 and G4. This is confirmed by the CDFs (Fig. 11) of the $Q$ index associated to the use of these sets of $C_{\mathrm{f}, \odot}$, where the dark green and cyan curves are mainly overlapped by the light green and blue distributions, respectively. The mean values of the $Q$ distribution for these cases are shown in Table 4. The smallest mean corresponds to the solar colors derived from the G5 set, something that, in principle, we might expect, because the stars contained in this group are, according to their stellar parameters, the most similar to the Sun.

Taking into account the previous discussion, we adopt $C_{\mathrm{f}, \odot}$ derived from the stars in the G5 group as our solar colors because this is the set that produces the smallest $Q$ index mean, that is, the smallest differences between the photospectra in J-PLUS and their spectroscopy counterparts in the literature. In Fig. C.1 we show a sample of 74 photospectra of asteroids in the MOOJa catalog, computed using our adopted colors. The quality of the photospectra and the improved resolution with respect to previous photometric surveys will let us perform taxonomical classifications for a large number of minor bodies.

However, we note that there exists a systematic reddening in the visible region of the resulting reflectance photospectra
Table 3. Asteroids within the MOOJa catalog for which there are published spectra or photospectra in different databases.

\begin{tabular}{|c|c|c|c|c|c|}
\hline Asteroid & Survey & $N_{\mathrm{f}}$ & $t_{\text {Total }}$ & Date & Time $^{(a)}$ \\
\hline 90 & $1,2,4,5$ & 10 & 35.2 & 2017-10-14 & $22: 31: 33$ \\
\hline 95 & $1,4,5,6$ & 10 & 35.3 & 2017-10-13 & $21: 15: 57$ \\
\hline 122 & 2,4 & 10 & 36.0 & 2017-09-14 & 01:00:37 \\
\hline 159 & 1,4 & 10 & 31.3 & 2017-11-21 & $21: 34: 23$ \\
\hline 184 & 4,5 & 12 & 38.0 & 2017-09-21 & $02: 55: 42$ \\
\hline 277 & 1 & 11 & 75.2 & 2017-08-14 & 02:19:06 \\
\hline 413 & 2,4 & 9 & 36.3 & 2017-03-29 & 20:08:07 \\
\hline 462 & 2,4 & 12 & 45.1 & 2017-11-26 & $20: 10: 27$ \\
\hline 712 & $1,2,4,6$ & 9 & 31.2 & $2017-10-15$ & $18: 55: 39$ \\
\hline 758 & 2 & 9 & 31.2 & 2017-11-21 & $23: 13: 51$ \\
\hline 784 & 4 & 12 & 39.4 & $2016-12-30$ & $02: 32: 55$ \\
\hline 851 & 1,3 & 10 & 38.9 & 2017-09-28 & $22: 13: 18$ \\
\hline 1024 & 4,5 & 12 & 64.2 & 2017-01-08 & 01:43:09 \\
\hline 1212 & $1,2,4$ & 12 & 37.9 & 2018-01-16 & $19: 24: 56$ \\
\hline 1245 & 1 & 12 & 38.0 & 2017-11-19 & $23: 51: 23$ \\
\hline 1677 & 5 & 12 & 43.9 & $2017-10-29$ & 02:19:01 \\
\hline 1796 & $1,4,5$ & 12 & 39.9 & 2017-08-22 & $01: 47: 24$ \\
\hline 1923 & 4,7 & 12 & 37.9 & 2017-09-20 & 00:00:53 \\
\hline 2149 & 3 & 12 & 38.8 & 2017-02-20 & $23: 08: 27$ \\
\hline 2251 & 4 & 12 & 39.9 & $2017-08-22$ & $02: 57: 29$ \\
\hline 2645 & 3 & 12 & 41.4 & 2017-10-27 & 04:20:00 \\
\hline 2730 & 4 & 12 & 38.1 & 2017-09-21 & $00: 10: 14$ \\
\hline 2902 & 4 & 12 & 37.9 & 2017-09-19 & $23: 11: 23$ \\
\hline 3259 & 5 & 12 & 41.1 & $2017-10-13$ & $18: 48: 10$ \\
\hline 3885 & 4 & 12 & 38.2 & $2017-11-21$ & $22: 22: 45$ \\
\hline 4733 & 4 & 12 & 39.7 & 2017-02-17 & $23: 55: 11$ \\
\hline 4993 & 4 & 12 & 38.2 & $2017-10-17$ & $00: 49: 08$ \\
\hline 6661 & 7 & 12 & 50.5 & 2017-08-15 & 03:09:08 \\
\hline 6769 & 7 & 12 & 38.1 & $2017-10-15$ & $00: 04: 47$ \\
\hline 7274 & 7 & 9 & 36.9 & 2016-12-07 & $02: 16: 50$ \\
\hline 34339 & 7 & 10 & 38.0 & 2017-09-24 & $03: 18: 31$ \\
\hline 85167 & 7 & 9 & 30.1 & 2017-01-07 & $00: 43: 29$ \\
\hline 113374 & 7 & 8 & 38.1 & 2017-09-23 & $22: 48: 49$ \\
\hline
\end{tabular}

Notes. ${ }^{(a)}$ The first column indicates the identification number of the asteroid, and the second column shows the survey(s) where the asteroid was observed. The numbers correspond to: 1-ECAS, 2-24CAS, 3SMASSI, 4-SMASSII, 5-S3OS2. 6-Sawyer, and 7-PRIMASS. The third and fourth columns show, respectively, the number of J-PLUS filters in which the asteroid has been observed, and the time elapsed (in minutes) from the exposure in the first filter to the exposure in the last one. Columns five and six are the date and the time at mid-observation.

when using the theoretical solar colors. Although this has been investigated, we did not find any satisfactory explanation for this effect. The mean reflectance variations that are induced, depending on which solar colors are used to compute the photospectra (2000 ASTM E-490 as the best theoretical choice, G5 stellar group as the empirical equivalent) are as follows: gSDSS, $0.0153 \pm 0.0013$; rSDSS, $0.042 \pm 0.003$; J0660, $0.047 \pm 0.005 ;$ iSDSS, $0.099 \pm 0.012 ; \quad J 0861,0.102 \pm 0.011$; zSDSS, $0.101 \pm 0.015$ (all in magnitude units).

Given these mean variations, we should bear in mind that the choice of solar colors might affect the slope of some spectra enough to modify a future taxonomical classification. In order to assess how this would affect taxonomical comparisons, we selected a subset of photospectra from Appendix C that visually match the templates of an S- (24 objects) and a V-type (4 objects), as these are the easiest ones to classify through 


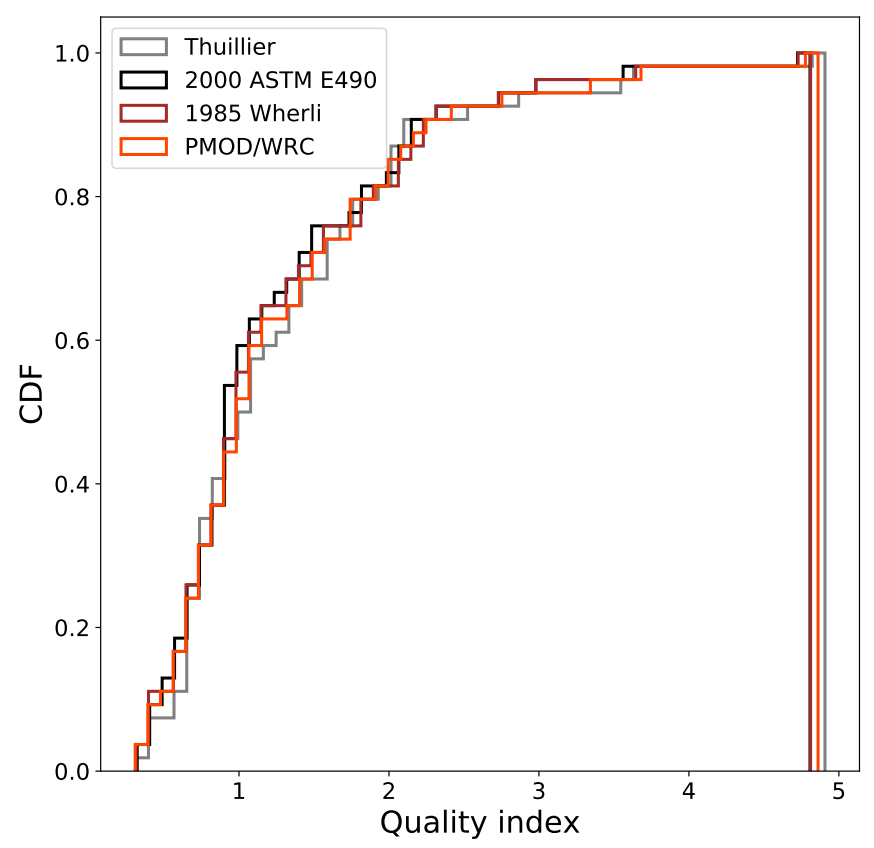

Fig. 10. Empirical cumulative distribution functions of the $Q$ index obtained after the comparison of the J-PLUS spectrophotometric data with the literature spectra, for the four different solar color choices.

Table 4. Mean values and their corresponding sigmas for the $Q$ distributions derived from the use of solar colors computed from Sun spectra (theoretical) and also from the ones obtained after computing the mean colors of different datasets of J-PLUS Sun-like stars (empirical).

\begin{tabular}{lcc}
\hline \hline Spectrum/ & $\bar{Q}$ & \\
Dataset & $\sigma_{Q}$ \\
\hline Theoretical & & \\
\hline 1985 Wehrli & 1.31 & 0.81 \\
2000 ASTM E-490 & 1.27 & 0.80 \\
Thuillier & 1.36 & 0.83 \\
PMOD/WRC & 1.33 & 0.83 \\
\hline Empirical & & \\
\hline G1 & 1.27 & 0.77 \\
G2 & 1.28 & 0.77 \\
G3 & 1.28 & 0.82 \\
G4 & 1.28 & 0.82 \\
G5 & 1.16 & 0.73 \\
\hline
\end{tabular}

means of visual inspection ${ }^{10}$. We then computed the mean reflectance photospectra for both cases using the 2000 ASTM E-490 and the G5 solar colors, and compared them to the corresponding taxonomic class templates (S- and V-types). In Fig. 12 we can see this systematic difference. Judging by the excessive reddening produced in both cases when using the theoretical colors compared to the corresponding taxonomic template, we are inclined to opt for the empirical colors derived from the G5 set as the optimal choice, as mentioned above.

Nevertheless, we stress that this selection was based on the analysis shown in the present work, and that a perfect choice

\footnotetext{
10 The objects that are unambiguosly matched by visual inspection to S-types are 473, 1608, 1677, 1736, 2149, 2498, 2902, 2963, 3259, 3338, 3446, 3863, 4028, 4109, 4703, 5293, 5795, 15419, 18960, 25046, 27082, 46304, 67611, and 102120; V-types are 1709, 2557, 9755, and 18195 .
}

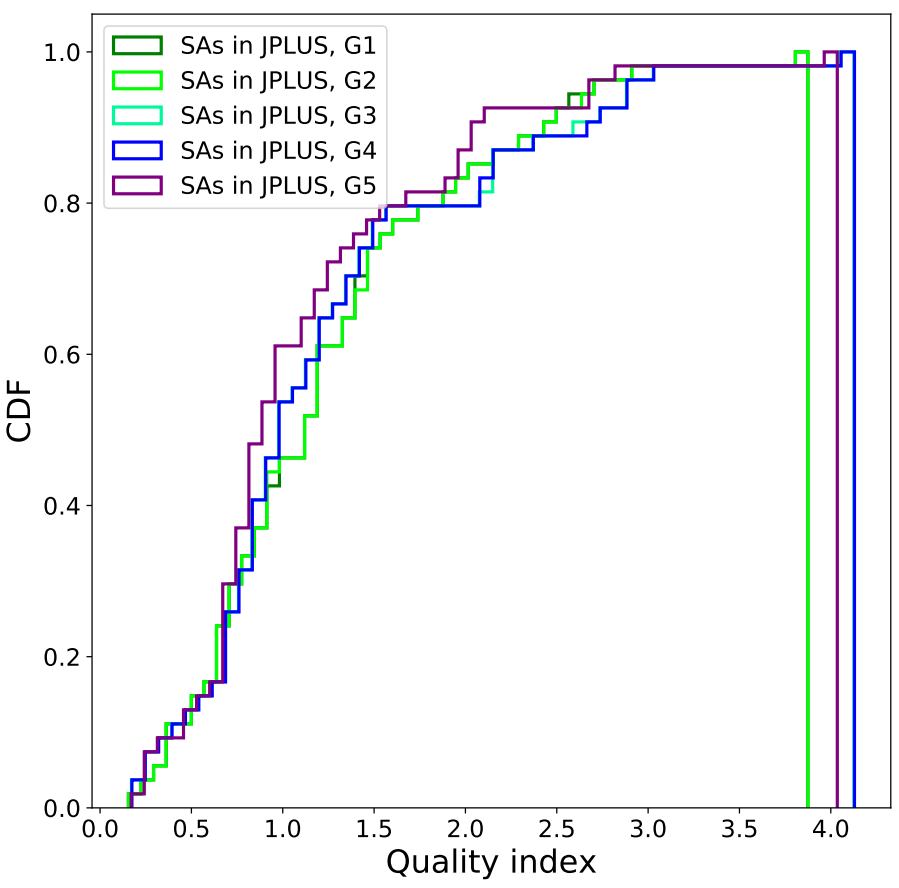

Fig. 11. Empirical CDFs of the $Q$ index obtained after the comparison of the J-PLUS spectrophotometric data with the literature spectra, for the five different solar color choices.

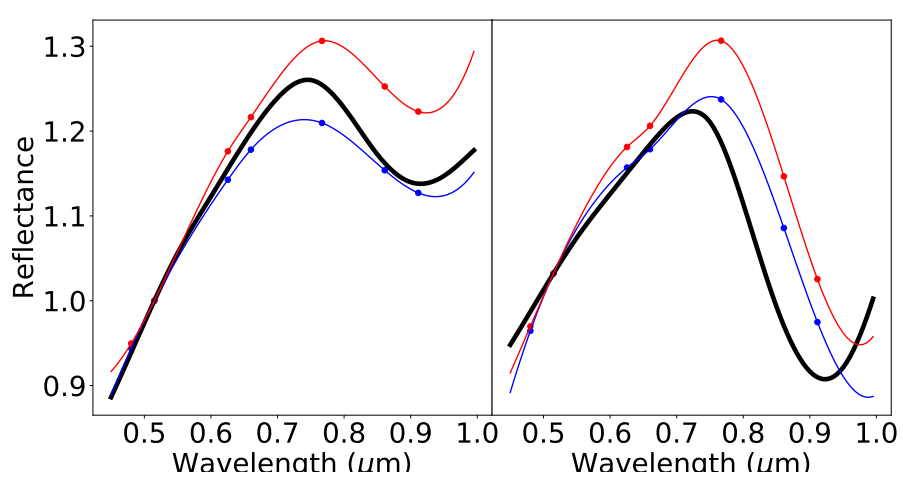

Fig. 12. Mean photospectra for a subset of S-types (left) and V-types (right) from Appendix C (see main text). The blue dots and curves represent the photospectra (reflectance points and a cubic spline fit to these points, respectively) computed using the empirical solar colors (G5 set). In red, the results corresponding to the use of theoretical solar colors (2000 ASTM E-490 set). The black curves represent the Bus-DeMeo template for an S-type (left) and a V-type (right).

of solar colors for the J-PLUS filter system does not exist. For this reason, all the solar colors that we computed in this work are presented in Table 2, leaving the final choice to the users of the catalog. In addition, future data releases might provide larger datasets of solar-type stars and better statistics, and thorough searches for solar analogs might alleviate the need for statistical analyses. We therefore encourage the reader and the catalog users to test other paths and, if possible, to improve the solar color computation.

\section{Caveats, issues, and possible solutions}

We stress that the spectrophotometry that can be generated using the MOOJa catalog is not exactly equivalent to the spectra that the observed asteroids would present if observed by 


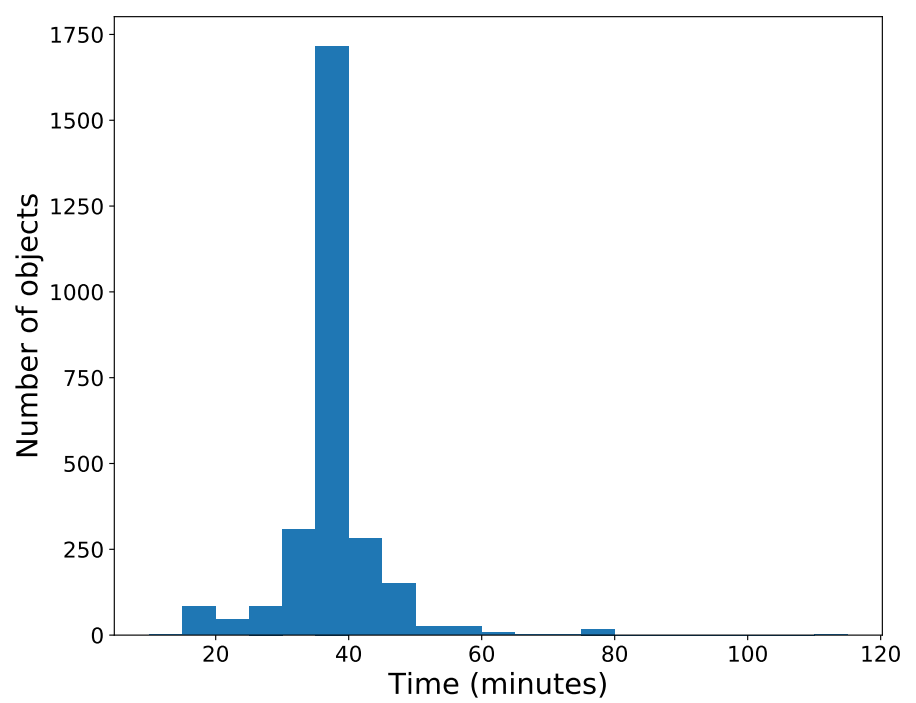

Fig. 13. Histogram showing the distribution of the exposure times, in minutes, for the asteroids observed in five or more filters. Bin size is $5 \mathrm{~min}$. We note that, in some cases, the total exposure time is longer than $60 \mathrm{~min}$. This is due to the choice of the optimal subset of observations for every asteroid (see Sect. 2). However, the majority of the cases are concentrated around the mean value.

a spectrograph. Usually, when observing a fixed object in the sky, such as a star, obtaining its spectrum or its photospectrum is essentially equivalent, as observations are often carried out on significantly shorter timescales than the typical variability timescale of most stars. The same is not true for asteroids: minor bodies are normally nonspherical objects, and might present different surface compositions from one region of the asteroid to another. Because of this, and their rotation, which is generally on the order of a few hours, the reflected sunlight varies in intensity along the observation time. If we obtain the spectrum (or photospectrum) of an asteroid using a short enough total exposure time, these fluctuations can be neglected. However, for long exposure times, and depending on the rotation period, shape, and pole orientation of the specific object, the induced reflectance variations might need to be taken into account.

Usually, we refer to exposure times as the total time elapsed from the opening of the shutter of the camera until the moment that the shutter closes; this is, the duration of one exposure. However, because in the case of the MOOJa catalog we are dealing with photometric spectra, we refer to exposure times as the total time elapsed from the first filter observation to the last, whether the asteroid was observed in the 12 filters or not. In Fig. 13, we show the total exposure times for all the asteroids observed within the MOOJa catalog. Although some asteroids present more than one hour of total exposure time (given the constraints that we imposed to obtain the spectrophotometry), in the majority of the cases the exposure time is around $40 \mathrm{~min}$, because of the filter observation sequence and the survey strategy.

The magnitude variation during this interval can be estimated by considering the light curves reported by the Asteroid Lightcurve Database ${ }^{11}$. The median rotation period for more than 18000 asteroids is $\sim 6.3 \mathrm{~h}$, and the median of the light curve amplitudes is $\sim 0.38 \mathrm{mag}$. Thus, for a light-curve amplitude of $0.38 \mathrm{mag}$ with a $6.3 \mathrm{~h}$ period, and a $40 \mathrm{~min}$ interval between the first and last filter measurement, we could estimate a mean

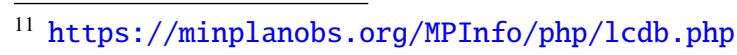

uncertainty of $\sim 0.16 \mathrm{mag}$, which might induce errors of around $15 \%$ when translated to reflectance.

Also, the fact that the filter measurements were not ordered according to the central wavelengths of the filters, as explained in Sect. 2, might introduce non-negligible uncertainties between adjacent points, depending on the time intervals between these contiguous spectrophotometric points (see Fig. 14). In Table 5 we show the estimated mean magnitude variations that might be induced by these time separations. We note that the higher estimated variations are those related to the 'jump' from filters $J 0861$ to $z$, and from filters $J 0660$ to $i$. This is evident when looking at Fig. B.1, for example, for asteroids 462, 1245, 1677, or 2251, where the points corresponding to the $z$ filter are shifted upwards with respect to the $J 0861$ points, or the $i$ point is shifted downwards with respect to the $J 0660$ point.

It is worth mentioning that, in some of the presented plots in Appendices B and C, a number of low-quality observations seem to persist: asteroid 851 , which shows a very red slope; 2730 , where there is a point that seems to lie far from the overall trend; or 6661, 6769, and 9688, which seem to have a very low $\mathrm{S} / \mathrm{N}$. In these cases, when the sigma-clipping algorithm was not enough to eliminate these datapoints, some additional procedures that could be used are, for example, filtering data that are highly discrepant with respect to the others (e.g., with respect to adjacent values, preceding and following) or highly discrepant with respect to the overall spectral shape (e.g., by fitting the spectra with an appropriate function and identifying outliers with respect to the trend). These options are left unexplored and open for future versions of the catalog.

We performed some simulations of the theoretical photospectra that would be obtained as a function of asteroid shape, aspect angle, rotational period, and superficial composition in order to assess how rotation could affect the obtained reflectance spectra of asteroids observed by J-PLUS. We consider asteroid shape models as sets of triangular facets. The bidirectional reflectance of each facet is obtained using the isotropic multiple scattering approximation (IMSA) equation (Hapke 2005) using a double-lobed Henyey-Greenstein phase function, with the single scatter albedo and phase parameters calculated using geometrical optics from sets of optical constants that vary with wavelength. The integrated reflectance of all facets that are visible and illuminated at a given observational geometry are then calculated for an array of wavelengths, which are then convolved with the band pass of each J-PLUS filter according to the filter sequence and using exposure times that are consistent with the observations.

Figure 15 shows the simulated light curve for an asteroid with spheroidal shape, an axis ratio of 0.7 , a rotation period of $6 \mathrm{~h}$, and an amplitude of $0.15 \mathrm{mag}$. Using optical constants for the HED meteorite Allan Hills A76005 (Davalos et al. 2017) and the Taggish Lake meteorite (Roush 2003), we simulated a $\mathrm{V}$ - and a D-type asteroid, respectively. The measured photospectrum would vary depending on the portion of the light curve that is observed (see Fig. 16).

As expected, the changes induced by rotation in the photospectrum are a function of the amplitude of the portion of the light curve sampled during the observations. The simulated light curve has a moderate amplitude $(\sim 0.15)$; if the amplitude were wider (i.e., if the object had a more elongated shape) this variation would be greater, enough to modify spectral points even with small errors (as we see in Figs. B.1 and C.1). In addition, given that these simulations were performed for two extreme taxonomical classes, such as a V- and a D-type, we expect this effect to induce sufficient changes to blur the limits 

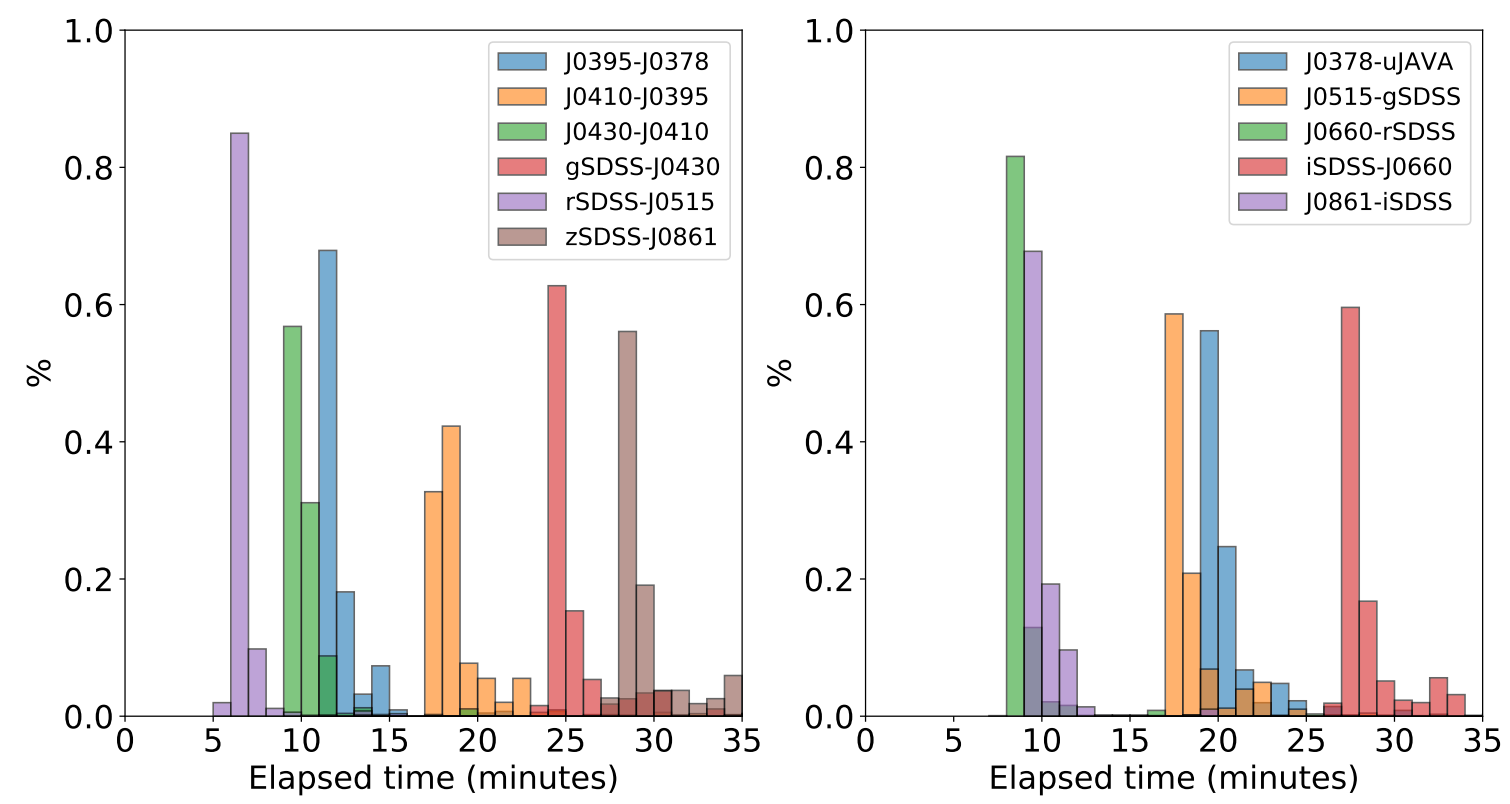

Fig. 14. Normalized histograms showing the distribution of elapsed times, in minutes, between contiguous filters. Usually, observations in filters at longer wavelengths come (although not immediately) after those at shorter wavelengths, except for the cases of filters $J 0410-J 0395, J 0430$ $J 0410, J 0515-g$, and $i-J 0660$ (see the filter sequence in Sect. 2). Histograms have been overplotted and separated according to their mean times for a better visual representation. Bin size is $1 \mathrm{~min}$.

Table 5. Mean values and their corresponding errors, in minutes, for the elapsed time between observations corresponding to contiguous filters.

\begin{tabular}{lccc}
\hline \hline Contiguous filters & $t_{\text {cont }}(\min )$ & $\Delta$ Mag. & $R_{\text {err }}(\%)^{(a)}$ \\
\hline$J 0378-u$ & $20.7 \pm 2.4$ & 0.08 & 7 \\
$J 0395-J 0378$ & $12.3 \pm 2.1$ & 0.05 & 5 \\
$J 0410-J 0395$ & $19 \pm 3$ & 0.08 & 7 \\
$J 0430-J 0410$ & $10.4 \pm 1.3$ & 0.04 & 4 \\
$g-J 0430$ & $26 \pm 3$ & 0.10 & 9 \\
$J 0515-g$ & $19 \pm 3$ & 0.08 & 7 \\
$r-J 0515$ & $6.6 \pm 1.2$ & 0.03 & 3 \\
$J 0660-r$ & $8.8 \pm 2.1$ & 0.04 & 4 \\
$i-J 0660$ & $29 \pm 4$ & 0.12 & 11 \\
$J 0861-i$ & $10.3 \pm 1.4$ & 0.04 & 4 \\
$z-J 0861$ & $30 \pm 4$ & 0.12 & 11 \\
\hline
\end{tabular}

Notes. ${ }^{(a)}$ The penultimate column corresponds to the mean magnitude variations estimated using the median values of the light-curve amplitudes and periods in the Asteroid Lightcurve Database (see main text) The final column represents the percentage of error induced in the reflectance by the variations in magnitude.

between closer classes such as C-, B-, X-, or even S-type asteroids.

The approach that we propose to solve this issue is to take into account the three individual expositions for each filter, using the slopes of the variations in time as a sort of 'partial' light curve, in order to correct these rotation-induced magnitude shifts.

There are also situations in which the reflectance variations between adjacent points are extremely large. These cases probably arise from the fact that the asteroid is passing over, or near, a star in the field at the moment of one of the exposures, or that one of the frames (or all of them) present low-quality data. The sigma-clipping algorithm removed some of these outliers. However, even after processing the data, some extreme

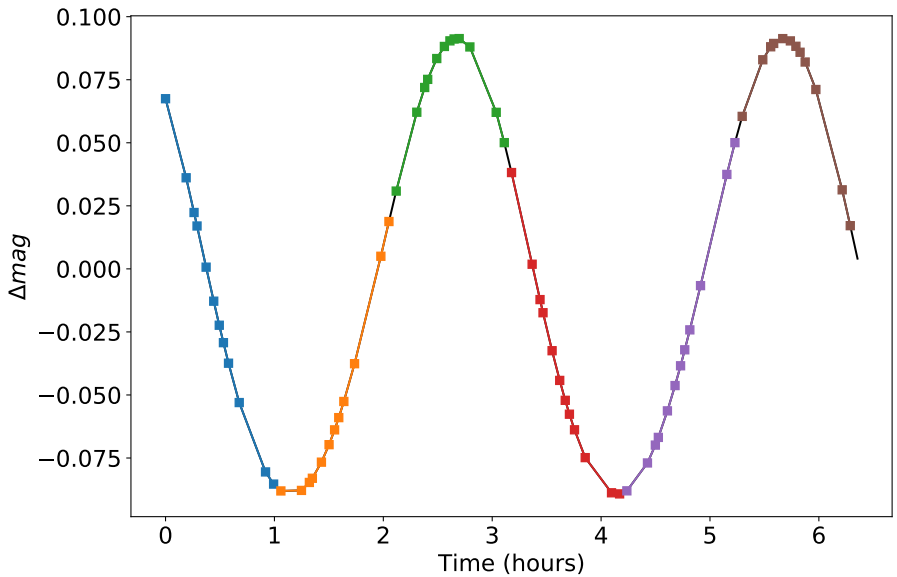

Fig. 15. Simulated light curve for a spheroid with an axial ratio of 0.7 , as observed by J-PLUS. Sets of points with the same color represent the central instant of observations with the 12 J-PLUS filters.

reflectance variations remain: in order to deal with this issue, the best approach would be to examine the three individual frames obtained for each filter, and to discard those in which the asteroid is located too close to a star in the background, or those in which the photometric quality is below some previously imposed threshold. Unfortunately, these individual frames were not available for the first data release, and therefore this issue will need to be addressed in a future version of the catalog.

\section{Summary and future work}

This work focuses on presenting the Moving Objects Observed from Javalambre (MOOJa) catalog to the scientific community. MOOJa is a compilation of spectrophotometric data for asteroids observed within the Javalambre Photometric Local Universe Survey in 12 filters covering wavelengths from 0.3 up to $1 \mu \mathrm{m}$. A total of 3666 different asteroids were detected. For this 


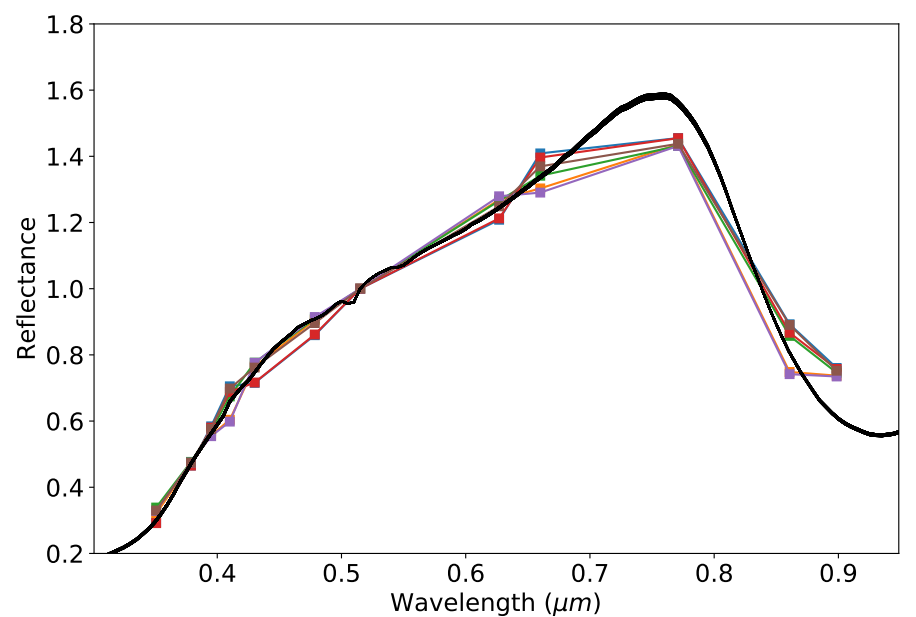

(a)

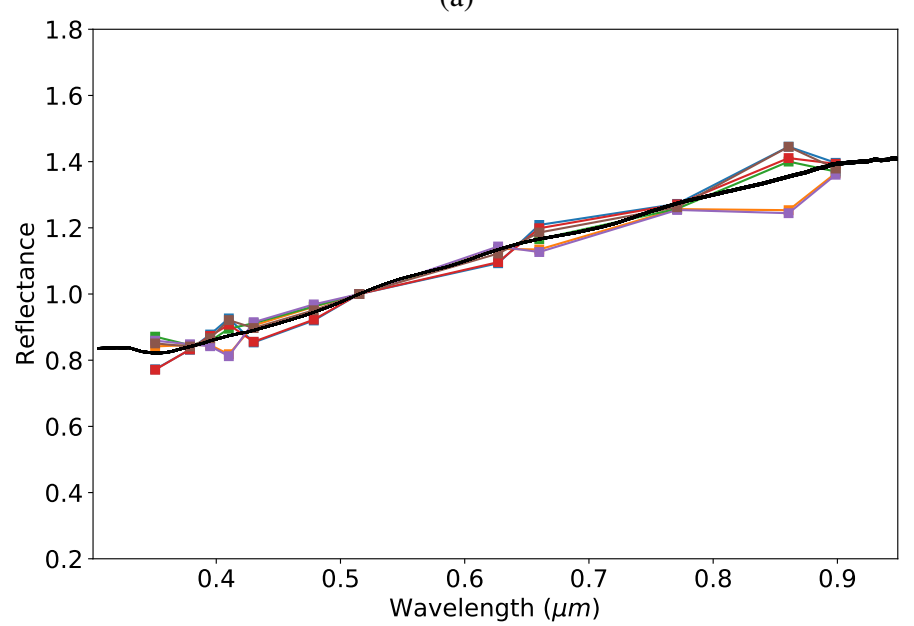

(b)

Fig. 16. (a) Resulting photospectra for the light curve in Fig. 15 using the optical constants of the HED meteorite Allan Hill A76005, with the reflectance spectra of the meteorite shown as a continuous black line. (b) Same as the previous figure, for the optical constants of the Taggish Lake meteorite.

initial collection of observations, we applied a sigma-clipping algorithm on the colors that are used to compute spectrophotometry, to clean and remove outliers, and to obtain a more robust dataset. The resulting color catalog that we provide consists of 3122 objects, of which 278 are observed in all 12 filters, that is, with a complete spectrum. The catalog was compiled using data only from the first data release of J-PLUS, which covers $\sim 10 \%$ of the sky region intended to be observed. We expect to recover around 18000 objects in the full duration of the survey, as well as to discover new ones, obtaining spectrophotometry for these bodies at the same time as their discovery.

We present a new method to select a suitable set of solar colors in the J-PLUS photometric system (from several options, computed both theoretically and empirically) that will be used in order to compute the photospectra of the asteroids within the MOOJa catalog. This method makes use of asteroids observed in past surveys and also observed by J-PLUS.

We also introduce the most relevant issues that might prevent us from maximizing the science outcome of the catalog: the most important one, the time interval between observations in contiguous filters; and secondly, flawed observations that cannot be removed with the sigma-clipping algorithm. We propose that the best course of action in order to remove both sources of error is to examine each of the three individual images in every filter: in the first case, correcting the magnitude variation induced by the light curve, and in the second, simply removing suboptimal exposures from the computations. Unfortunately, these data were not available as part of the DR1, but are available within the DR2.There is currently an ongoing effort to further extend the catalog, which will be updated once all the minor body data within the DR2 are recovered and processed.

In addition, we would like to address some interesting points that might serve as a checklist for future works:

- The first and most immediate analysis should focus on the taxonomic classification of the asteroids observed within the catalog. The fact that the observations are made using 12 filters will provide a huge number of asteroid classifications, and will exponentially increase our knowledge of the distribution of the different materials throughout the Solar System.

- As the number of UV filters in which asteroids are observed is higher than any previous survey in the field, this might provide new insights into this wavelength region. A novel taxonomic system in the UV should be probed, to enhance our knowledge of minor body science within this spectral domain.

- Given the spectral resolution and the distribution of the central wavelengths of the filters, the information on the MOOJa catalog will allow for a more robust definition of the $0.7 \mu \mathrm{m}$ absorption band (related to aqueous altered minerals on the surface of the asteroid) than using SDSS data.

We would like to mention that there exists another ongoing survey, S-PLUS (Southern Photometric Local Universe Survey, see Mendes de Oliveira et al. 2019) that is carried out using an identical duplicate of the telescope used in Javalambre, the T80, located near the summit of Cerro Tololo in central Chile. The aim of this survey is almost the same as J-PLUS, but instead of covering the sky of the northern hemisphere, will cover the southern side. This will provide a similar number of spectrophotometric data for asteroids.

Along these lines, another important survey that might benefit from J-PLUS operations (and viceversa) is the Gaia mission (see Mignard et al. 2007; Delbo' et al. 2012; Tanga \& Mignard 2012), because its spectrophotometric system covers almost the same region as J-PLUS. Specifically, the Gaia-BP spectrophotometer covers the UV region, where J-PLUS also offers a total of seven narrow-band and two broad-band filters. Thus, J-PLUS data might be used, for example, to test and calibrate Gaia spectra for minor bodies in the UV range. The synergies that might arise from combining both datasets are very interesting and should be taken into account.

In summary, the vast number of photospectra for minor bodies that this catalog and its future versions will provide might be the first step in a new era in which large-area surveys, will be key to solving the unanswered questions surrounding the minor bodies of the Solar System thanks to the wealth of data (and its quality) that will be available to the community.

Acknowledgements. DM acknowledges the CNPq for the support in form of a PCI grant, and also the FAPERJ for the support in the form of a Pós-Doutorado Nota 10 grant. JMC acknowledges CPNq for support through a research fellowship. AAC acknowledges support from FAPERJ (grant E26/203.186/2016) and CNPq (grants 304971/2016-2 and 401669/2016-5), from the Universidad de Alicante under contract UATALENTO18-02, and from the State Agency for Research of the Spanish MCIU through the "Center of Excellence Severo Ochoa" award to the Instituto de Astrofísica de Andalucía (SEV-2017-0709) MDP ackowledges funding by the Preeminent Postdoctoral Program (P3) at the University of Central Florida. JL acknowledges support from the project 
AYA2015-67772-R (MINECO). AG acknowledges CAPES for the support in the form of a Phd. grant. MM acknowledges funding by the European Space Agency under the research contract C4000122918. ESM and FJE acknowledge funding by the Spanish State Research Agency (AEI) Projects AYA201784089 and MDM-2017-0737 at Centro de Astrobiología (CSIC-INTA), Unidad de Excelencia María de Maeztu. The present work is based on observations made with the JAST/T80 telescope for the J-PLUS project at the Observatorio Astrofísico de Javalambre in Teruel, a Spanish Infraestructura Cientifico-Técnica Singular (ICTS) owned, managed and operated by the Centro de Estudios de Física del Cosmos de Aragón (CEFCA). Data has been processed and provided by CEFCA's Unit of Processing and Archiving Data (UPAD). Funding for the J-PLUS Project has been provided by the Governments of Spain and Aragón through the Fondo de Inversiones de Teruel; the Aragón Governmen through the Research Groups E96, E103, and E16_17R; the Spanish Ministry of Science, Innovation and Universities (MCIU/AEI/FEDER, UE) with grant PGC2018-097585-B-C21 and PGC2018-097585-B-C22; the Spanish Ministry of Economy and Competitiveness (MINECO) under AYA2015-66211-C2-1P, AYA2015-66211-C2-2, AYA2012-30789, and ICTS-2009-14; and European FEDER funding (FCDD10-4E-867, FCDD13-4E-2685). The Brazilian agencies FAPESP and the National Observatory of Brazil have also contributed to this project.

\section{References}

Alí-Lagoa, V., de León, J., Licandro, J., et al. 2013, A\&A, 554, A71

Berthier, J., Vachier, F., Thuillot, W., et al. 2006, in SkyBoT, a New VO Service to Identify Solar System Objects, eds. C. Gabriel, C. Arviset, D. Ponz, \& S. Enrique, ASP Conf. Ser., 351, 367

Bertin, E. 2006, in Automatic Astrometric and Photometric Calibration with SCAMP, eds. C. Gabriel, C. Arviset, D. Ponz, \& S. Enrique, ASP Conf. Ser. 351,112

Bertin, E., \& Arnouts, S. 1996, A\&AS, 117, 393

Binzel, R. P., DeMeo, F. E., Turtelboom, E. V., et al. 2019, Icarus, 324, 41

Breiman, L. 2001, Mach. Learn., 45, 5

Bus, S. J., \& Binzel, R. P. 2002a, Icarus, 158, 146

Bus, S. J., \& Binzel, R. P. 2002b, Icarus, 158, 106

Carvano, J. M., Hasselmann, P. H., Lazzaro, D., \& Mothé-Diniz, T. 2010, A\&A, 510, A43

Cenarro, A. J., Moles, M., Marín-Franch, A., et al. 2014, Proc. SPIE, 9149, 91491I

Cenarro, A. J., Moles, M., Cristóbal-Hornillos, D., et al. 2019, A\&A, 622, A176

Chance, K., \& Kurucz, R. L. 2010, J. Quant. Spectrosc. Radiat. Transf., 111, 1289

Chapman, C. R., Gaffey, M., \& McFadden, L. 2005, NASA Planetary Data System, EAR

Davalos, J. A. G., Carvano, J. M., \& Blanco, J. 2017, Icarus, 285, 275

de León, J., Campins, H., Morate, D., et al. 2018, Icarus, 313, 25

de Leon, J., Pinilla-Alonso, N., Campins, H., et al. 2018, AAS/Division for Planetary Sciences Meeting Abstracts \#50, 310.05

Delbo', M., Gayon-Markt, J., Busso, G., et al. 2012, Planet. Space Sci., 73, 86

DeMeo, F. E., \& Carry, B. 2014, Nature, 505, 629

DeMeo, F. E., Binzel, R. P., Slivan, S. M., \& Bus, S. J. 2009, Icarus, 202, 160

Fröhlich, C., \& Lean, J. 1997, in Correlated Phenomena at the Sun, in the Heliosphere and in Geospace, ed. A. Wilson, ESA SP, 415, 227
Haberreiter, M., Schöll, M., Dudok de Wit, T., et al. 2017, J. Geophys. Res.: Space Phys., 122, 5910

Hapke, B. 2005, Theory of Reflectance and Emittance Spectroscopy (Cambridge: Cambridge University Press)

Hasselmann, P. H., Carvano, J. M., \& Lazzaro, D. 2011, NASA Planetary Data System, 145

Hasselmann, P. H., Fulchignoni, M., Carvano, J. M., Lazzaro, D., \& Barucci, M. A. 2015, A\&A, 577, A147

Ishihara, D., Onaka, T., Kataza, H., et al. 2010, A\&A, 514, A1

Ivezić, Ž., Tabachnik, S., Rafikov, R., et al. 2001, AJ, 122, 2749

Ivezić, Ž., Lupton, R. H., Jurić, M., et al. 2002, AJ, 124, 2943

Kaiser, N., Burgett, W., Chambers, K., et al. 2010, Proc. SPIE, 7733, 77330E

Lawrence, A., Warren, S. J., Almaini, O., et al. 2007, MNRAS, 379, 1599

Lazzaro, D., Angeli, C. A., Carvano, J. M., et al. 2004, Icarus, 172, 179

Lazzaro, D., Barucci, M. A., Perna, D., et al. 2013, A\&A, 549, L2

Licandro, J., Popescu, M., Morate, D., \& de León, J. 2017, A\&A, 600, A126

Logroño-García, R., Vilella-Rojo, G., López-Sanjuan, C., et al. 2019, A\&A, 622, A 180

López-Sanjuan, C., Varela, J., Cristóbal-Hornillos, D., et al. 2019, A\&A, 631, A119

Mahlke, M., Solano, E., Bouy, H., et al. 2019, Astron. Comput., 28, 100289

Mainzer, A., Grav, T., Bauer, J., et al. 2011, ApJ, 743, 156

Mainzer, A., Bauer, J., Cutri, R. M., et al. 2014, ApJ, 792, 30

Marin-Franch, A., Taylor, K., Cenarro, J., Cristobal-Hornillos, D., \& Moles, M. 2015, IAU Gen. Assem., 29, 2257381

Masiero, J. R., Mainzer, A. K., Grav, T., et al. 2011, ApJ, 741, 68

Masiero, J. R., Mainzer, A. K., Bauer, J. M., et al. 2013, ApJ, 770, 7

Masiero, J. R., Grav, T., Mainzer, A. K., et al. 2014, ApJ, 791, 121

Mendes de Oliveira, C., Ribeiro, T., Schoenell, W., et al. 2019, MNRAS, 489, 241

Mignard, F., Cellino, A., Muinonen, K., et al. 2007, Earth Moon Planets, 101, 97

Morate, D., Licandro, J., Popescu, M., \& de León, J. 2018, A\&A, 617, A72

Parker, A., Ivezić, Ž., Jurić, M., et al. 2008, Icarus, 198, 138

Popescu, M., Licandro, J., Morate, D., et al. 2016, A\&A, 591, A115

Popescu, M., Licandro, J., Carvano, J. M. F., et al. 2018, A\&A, 617, A12

Rivkin, A. S. 2012, Icarus, 221, 744

Roush, T. L. 2003, Meteor. Planet. Sci., 38, 419

Sawyer, S. 2005, NASA Planetary Data System, 31

Skrutskie, M. F., Cutri, R. M., Stiening, R., et al. 2006, AJ, 131, 1163

Smalley, B. 2005, Mem. Soc. Astron. It. Suppl., 8, 130

Soderblom, D. R., \& King, J. R. 1998, in Solar Analogs: Characteristics and Optimum Candidates, ed. J. C. Hall, 41

Solano, E., Martín, E. L., Caballero, J. A., et al. 2019, A\&A, 627, A29

Sutherland, W., Emerson, J., Dalton, G., et al. 2015, A\&A, 575, A25

Tanga, P., \& Mignard, F. 2012, Planet. Space Sci., 73, 5

Thuillier, G., Hersé, M. Labs, D., et al. 2003, Sol. Phys., 214, 1

Thuillier, G., Floyd, L., Woods, T. N., et al, 2004, Solar Variability and its Effects on Climate. Geophysical Monograph 141, 141, 171

Wehrli, C. 1985, Publication no. 615, Physikalisch-Meteorologisches Observatorium + World Radiation Center (PMO/WRC) Davos Dorf

Whitten, D. D., Placco, V. M., Beers, T. C., et al. 2019, A\&A, 622, A182

Wright, E. L., Eisenhardt, P. R. M., Mainzer, A. K., et al. 2010, AJ, 140, 1868

Xu, S., Binzel, R. P., Burbine, T. H., \& Bus, S. J. 1995, Icarus, 115, 1

York, D. G., Adelman, J., Anderson, J. E., Jr., et al. 2000, AJ, 120, 1579

Zellner, B., Tholen, D. J., \& Tedesco, E. F. 1985, Icarus, 61, 355 


\section{Appendix A: Color vs. color examples}

Here we show four color-color distributions of asteroids observed within the MOOJa catalog. In gray, all the observed objects within the colors catalog (that are observed in the corresponding filters). Overplotted, the asteroids (only C-, S-, V-, and X-types) that were previously classified according to their
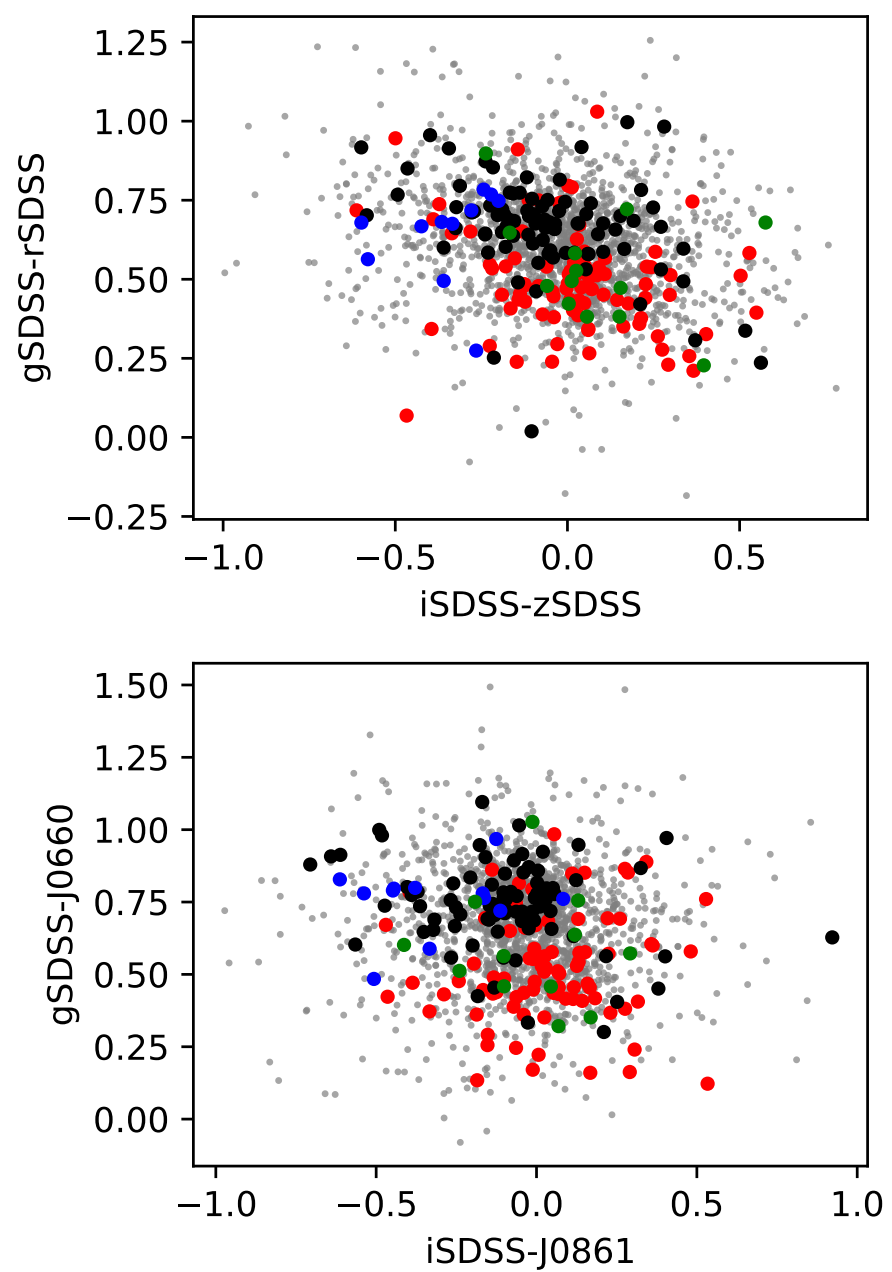

Fig. A.1. Color-color distributions for some filter combinations.
SDSS colors (see Carvano et al. 2010): C-types, in red; S-types, in black; V-types, in blue; X-types, in green. Top panels are a combination of broad-band-filters-only (left) and narrow-bandfilters-only (right). Bottom panels are combinations of both broad and narrow-band filters. It is easy to separate between, at least, $\mathrm{C}$ - and S-types using the combinations in the top- and bottom-left panels.
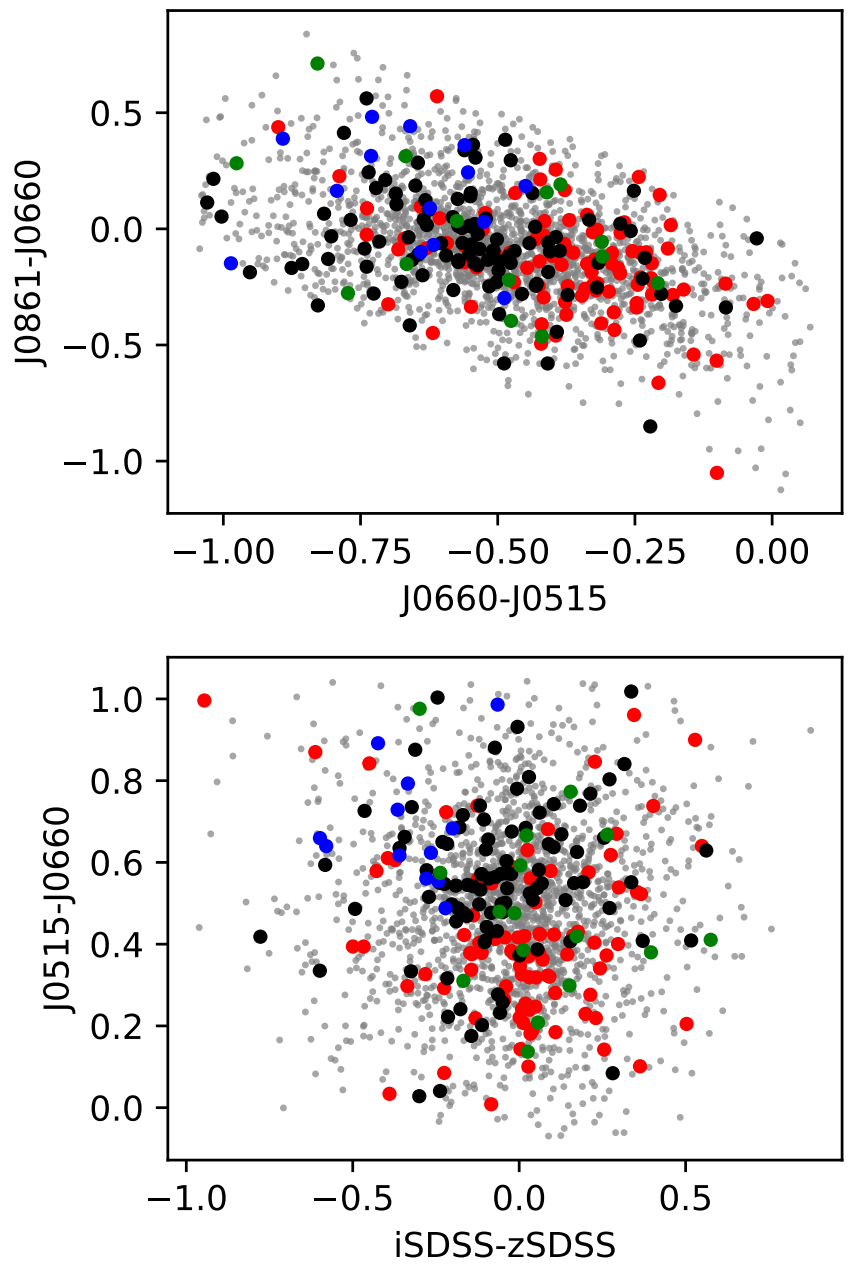


\section{Appendix B: J-PLUS spectra vs. literature spectra}

Photospectra in the $0.3-1.0 \mu \mathrm{m}$ region of the 33 asteroids within the MOOJa catalog which are also available in the literature. The J-PLUS spectrophotometry (computed using the G5 solar colors, see Sect. 3) is represented in blue, and the literature spectra, overplotted, are represented as follows: ECAS, orange right-pointing triangles, $\triangleright$; SMASSI, green circles, ○; SMAS-
SII, black squares, $\square$; S3OS2, magenta left-pointing triangles $\triangleleft$; 24CAS, gray down-pointing arrows $v$; Sawyer, cyan diamonds, $\diamond$; PRIMASS, red crosses, $\times$. All photospectra are normalized to $0.515 \mu \mathrm{m}$, which is the central wavelength of the $J 0515$ filter, which, among the central wavelengths of all the filters, is the nearest one to $0.55 \mu \mathrm{m}$, the value that is the most widely used to normalize the visible spectra of asteroids.
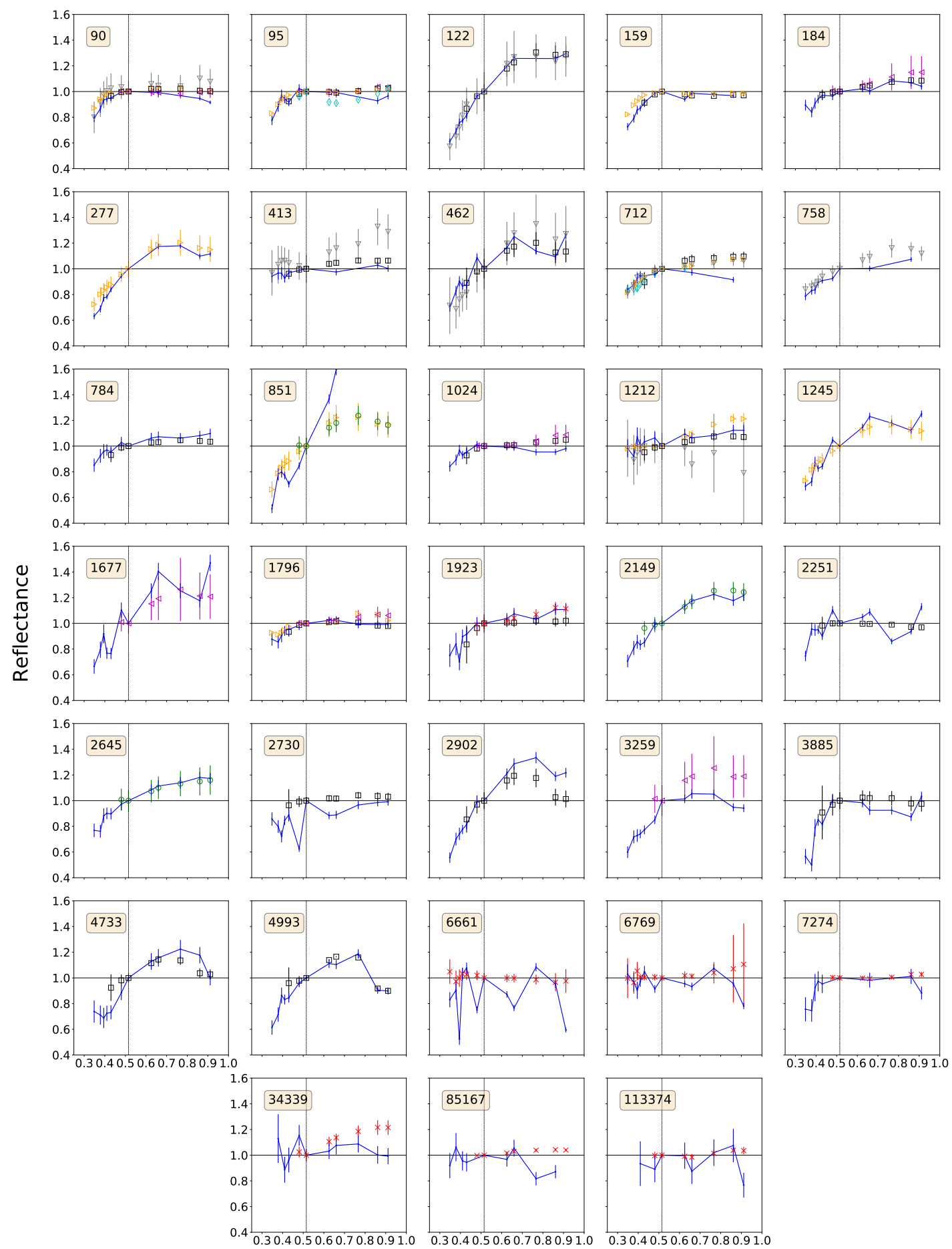

Wavelength $(\mu \mathrm{m})$

Fig. B.1. Comparison between photospectra of J-PLUS asteroids and visible literature spectra. 
D. Morate et al.: J-PLUS: A first glimpse at the spectrophotometry of asteroids. The MOOJa catalog

\section{Appendix C: J-PLUS photospectra collection}

Photospectra in the $0.3-1.0 \mu \mathrm{m}$ region of 74 asteroids within the MOOJa catalog. These objects were detected by J-PLUS in all
12 filters and with $\left(m_{f}-m_{J 0515}\right)_{e r r}<0.1$ magnitude units. The dots represent the J-PLUS data, and the solid curve is a cubic polynomial fit, for a better visualization of the overall spectral shape.

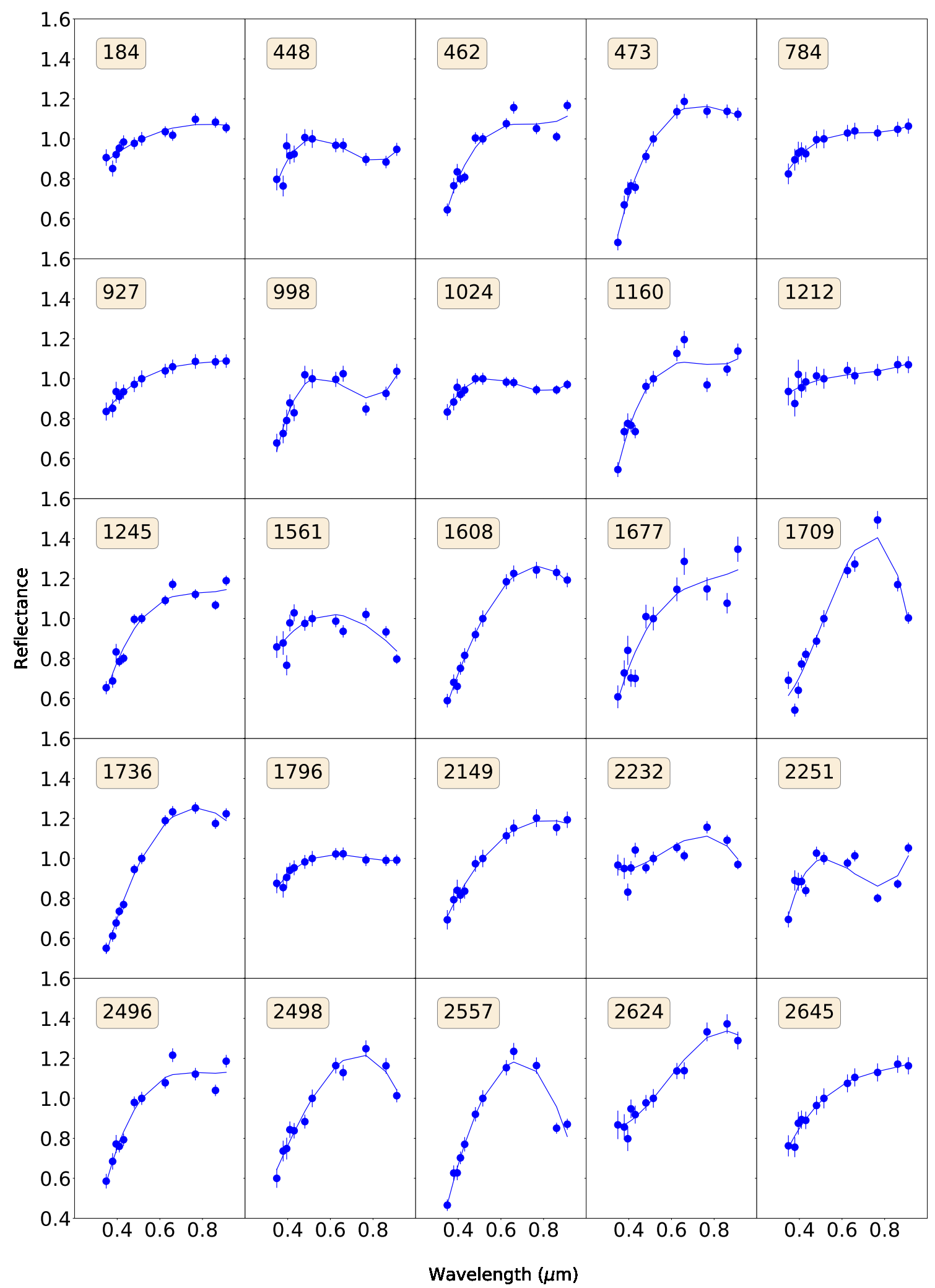

Fig. C.1. Photospectra of asteroids detected in the MOOJa catalog. 


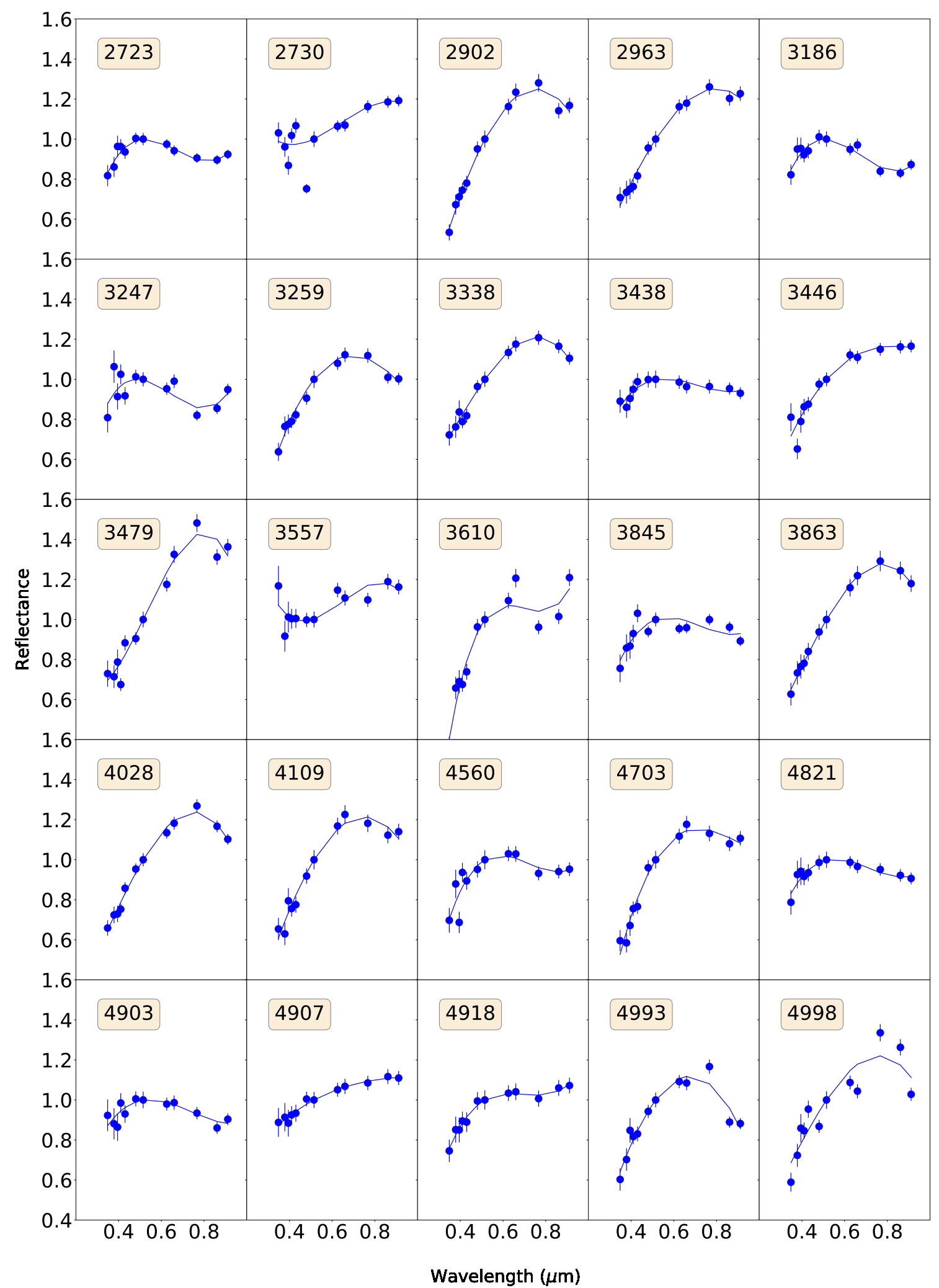

Fig. C.2. Photospectra of asteroids detected in the MOOJa catalog (continued). 
D. Morate et al.: J-PLUS: A first glimpse at the spectrophotometry of asteroids. The MOOJa catalog

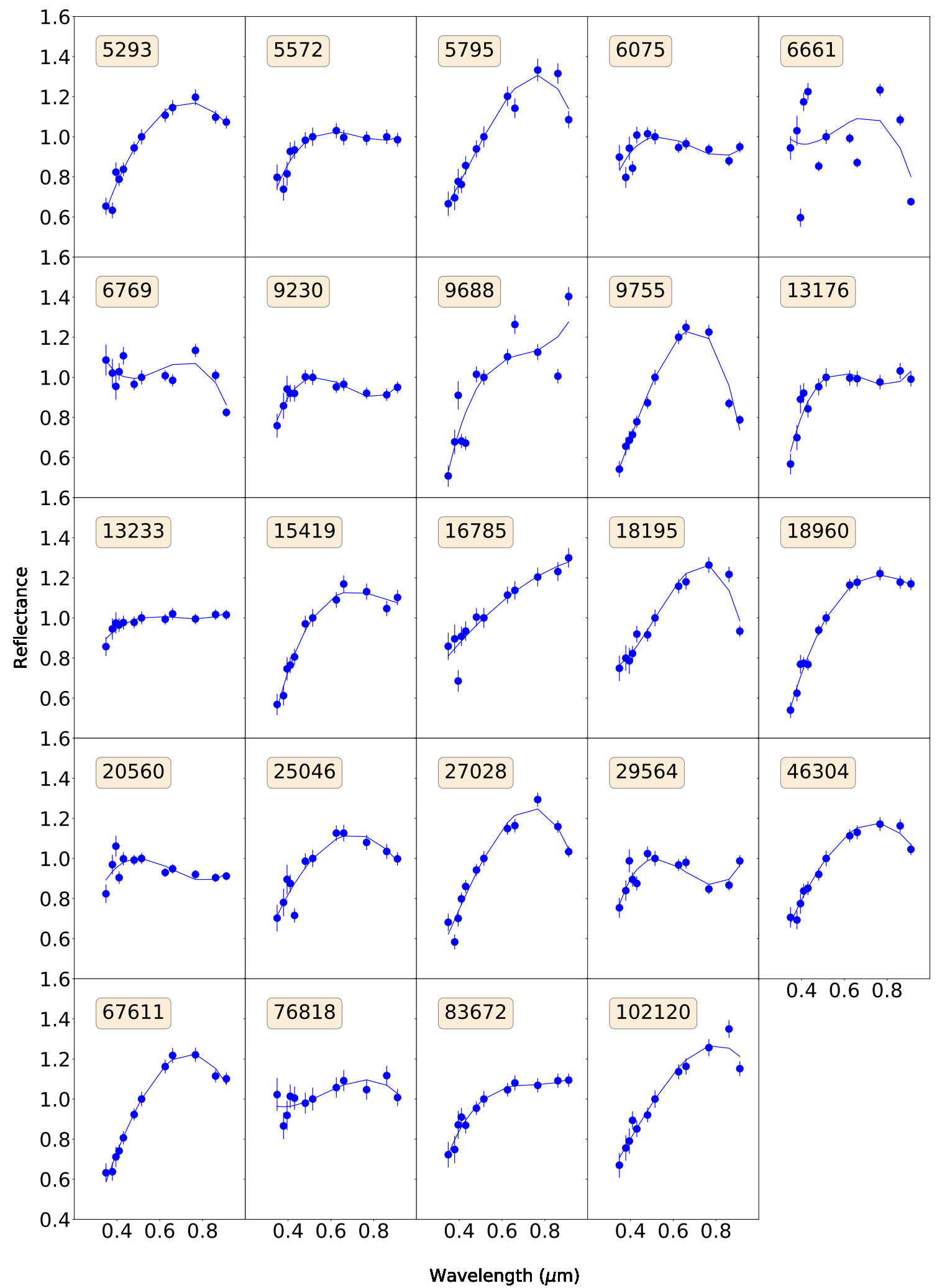

Fig. C.3. Photospectra of asteroids detected in the MOOJa catalog (continued). 